\title{
Long-time asymptotics of the Navier-Stokes and vorticity equations on $\mathbb{R}^{3}$
}

\author{
Thierry Gallay \\ Université de Paris-Sud \\ Mathématiques \\ Bâtiment 425 \\ F-91405 Orsay \\ France
}

\author{
C. Eugene Wayne \\ Department of Mathematics \\ and Center for BioDynamics \\ Boston University \\ 111 Cummington Street \\ Boston, MA 02215, USA
}

February 23, 2001

\begin{abstract}
We use the vorticity formulation to study the long-time behavior of solutions to the Navier-Stokes equation on $\mathbb{R}^{3}$. We assume that the initial vorticity is small and decays algebraically at infinity. After introducing self-similar variables, we compute the long-time asymptotics of the rescaled vorticity equation up to second order. Each term in the asymptotics is a self-similar divergence-free vector field with Gaussian decay at infinity, and the coefficients in the expansion can be determined by solving a finite system of ordinary differential equations. As a consequence of our results, we are able to characterize the set of solutions for which the velocity field satisfies $\|\mathbf{u}(\cdot, t)\|_{L^{2}}=o\left(t^{-5 / 4}\right)$ as $t \rightarrow+\infty$. In particular, we show that these solutions lie on a smooth invariant submanifold of codimension 11 in our function space.
\end{abstract}

\section{Introduction}

We consider the motion of an incompressible viscous fluid filling the whole space $\mathbb{R}^{3}$. If no external force is applied, the velocity $\mathbf{u}(x, t)$ of the fluid satisfies the Navier-Stokes equation

$$
\partial_{t} \mathbf{u}+(\mathbf{u} \cdot \nabla) \mathbf{u}=\nu \Delta \mathbf{u}-\frac{1}{\rho} \nabla p, \quad \operatorname{div} \mathbf{u}=0,
$$

where $\rho$ is the density of the fluid, $\nu$ is the kinematic viscosity, and $p(x, t)$ is the pressure field. Replacing $x, t, \mathbf{u}, p$ with the dimensionless quantities

$$
\frac{x}{L}, \quad \frac{\nu t}{L^{2}}, \frac{L \mathbf{u}}{\nu}, \frac{L^{2} p}{\rho \nu^{2}},
$$

where $L$ is an arbitrary length scale, Eq.(11) is transformed into

$$
\partial_{t} \mathbf{u}+(\mathbf{u} \cdot \nabla) \mathbf{u}=\Delta \mathbf{u}-\nabla p, \quad \operatorname{div} \mathbf{u}=0 .
$$


Since the length $L$ was arbitrary, Eq.(2) is still invariant under the scaling transformation

$$
\mathbf{u}(x, t) \mapsto \lambda \mathbf{u}\left(\lambda x, \lambda^{2} t\right), \quad p(x, t) \mapsto \lambda^{2} p\left(\lambda x, \lambda^{2} t\right)
$$

for any $\lambda>0$.

As no external force is applied, it is intuitively clear that all finite-energy solutions of (2) should converge, as time goes to infinity, to the rest state $\mathbf{u} \equiv 0, p \equiv$ const. As a matter of fact, if $\mathbf{u}(x, t)$ is any global weak solution in $L^{2}\left(\mathbb{R}^{3}\right)$ satisfying the energy inequality, it is known that $\|\mathbf{u}(\cdot, t)\|_{L^{2}} \rightarrow 0$ as $t \rightarrow \infty$ (see Wiegner [24]). Moreover, if

$$
\left\|\mathrm{e}^{t \Delta} \mathbf{u}(\cdot, 0)\right\|_{L^{2}} \leq \frac{C}{(1+t)^{\alpha}}, \quad t \geq 0
$$

for some $\alpha \geq 0$, then

$$
\|\mathbf{u}(\cdot, t)\|_{L^{2}} \leq \frac{C^{\prime}}{(1+t)^{\beta}}, \quad t \geq 0
$$

where $\beta=\min (\alpha, 5 / 4)$. This result shows that the solutions of (2) decay to zero at the same rate as those of the linear heat equation, provided this rate does not exceed $t^{-5 / 4}$. As we shall see below, the restriction $\beta \leq 5 / 4$ in (5) is due to the nonlinearity in (2) and to the incompressibility condition $\operatorname{div} \mathbf{u}=0$.

Wiegner's result raises a very natural question: can we characterize the set of solutions of (2) such that $t^{5 / 4}\|\mathbf{u}(\cdot, t)\|_{L^{2}} \rightarrow 0$ as $t \rightarrow \infty$ ? Put differently, given a solution $\mathbf{u}(x, t)$ satisfying (5) with $\beta=5 / 4$, under which conditions can we prove the corresponding lower bound $\|\mathbf{u}(\cdot, t)\|_{L^{2}} \geq C(1+t)^{-5 / 4}$ ? This problem has been intensively studied during the last 15 years, especially by M.E. Schonbek [18], [19], 20], [21], who found sufficient conditions for such a lower bound to hold. For technical reasons, these results were established assuming some additional decay of the initial data $\mathbf{u}_{0}=\mathbf{u}(\cdot, 0)$. Typically, it was assumed that $\mathbf{u}_{0} \in L^{2}\left(\mathbb{R}^{3}\right)^{3}$ and $(1+|x|) \mathbf{u}_{0} \in L^{1}\left(\mathbb{R}^{3}\right)^{3}$, so that (四) holds with $\alpha=5 / 4$.

Very recently, T. Miyakawa and M.E. Schonbek obtained an interesting characterization of the "rapidly decreasing" solutions of the Navier-Stokes equation in $\mathbb{R}^{N}, N \geq 2$. In the case $N=3$, their result reads:

Theorem 1.1 [17] Assume that $\mathbf{u}_{0} \in L^{2}\left(\mathbb{R}^{3}\right)^{3}$, div $\mathbf{u}_{0}=0$, and $(1+|x|) \mathbf{u}_{0} \in L^{1}\left(\mathbb{R}^{3}\right)^{3}$. Let $\mathbf{u}(x, t)$ be a global weak solution of (可) with initial data $\mathbf{u}(\cdot, 0)=\mathbf{u}_{0}$, satisfying the bound (5) with $\beta=5 / 4$. For all $k, \ell \in\{1,2,3\}$, define

$$
b_{k \ell}=\int_{\mathbb{R}^{3}} x_{k} u_{\ell}(x, 0) \mathrm{d} x, \quad c_{k \ell}=\int_{0}^{\infty} \int_{\mathbb{R}^{3}} u_{k}(x, t) u_{\ell}(x, t) \mathrm{d} x \mathrm{~d} t .
$$

Then

$$
\lim _{t \rightarrow \infty} t^{5 / 4}\|\mathbf{u}(\cdot, t)\|_{L^{2}}=0
$$

if and only if there exists $c \geq 0$ such that

$$
b_{k \ell}=0 \quad \text { and } \quad c_{k \ell}=c \delta_{k \ell}, \quad k, \ell \in\{1,2,3\} .
$$

The proof is a direct calculation using the integral equation satisfied by the solutions of (2). As the authors themselves remark, this argument does not provide much intuition as 
to the meaning of the conditions (8). From our point of view, the most surprising feature of Theorem 1.1 is the fact that assertion (7) is translation invariant in time, whereas conditions (8) are not. More precisely, if a solution $\mathbf{u}(x, t)$ satisfies (7), so does $\mathbf{u}(x, t+T)$ for any $T>0$. However, the property that $(1+|x|) \mathbf{u}$ be integrable is not preserved under the Navier-Stokes evolution (even for strong solutions, where uniqueness holds). Thus, if $(1+|x|) \mathbf{u}_{0} \in L^{1}\left(\mathbb{R}^{3}\right)^{3}$, then in general $(1+|x|) \mathbf{u}(\cdot, T) \notin L^{1}\left(\mathbb{R}^{3}\right)^{3}$ for $T>0$, so that the moments $b_{k \ell}$ do not make sense if $\mathbf{u}(x, 0)$ is replaced by $\mathbf{u}(x, T)$. Moreover, if the matrix $\left(c_{k \ell}\right)$ is scalar, this property will be lost if $\mathbf{u}(x, t)$ is replaced by $\mathbf{u}(x, t+T)$. Summarizing, Theorem 1.1 is a characterization of those rapidly decreasing solutions of (2) whose initial data lie in the noninvariant subspace $\left\{\mathbf{u}_{0} \in L^{2}\left(\mathbb{R}^{3}\right)^{3} \mid(1+|x|) \mathbf{u}_{0} \in L^{1}\left(\mathbb{R}^{3}\right)^{3}\right\}$. Nontrivial examples of such solutions have been recently constructed by L. Brandolese [1].

In this paper, we use the vorticity formulation to study the long-time behavior of the solutions of the Navier-Stokes equation (2). Setting $\boldsymbol{\omega}=\operatorname{rot} \mathbf{u}$, Eq.(2) is transformed into

$$
\partial_{t} \boldsymbol{\omega}+(\mathbf{u} \cdot \nabla) \boldsymbol{\omega}-(\boldsymbol{\omega} \cdot \nabla) \mathbf{u}=\Delta \boldsymbol{\omega}, \quad \operatorname{div} \boldsymbol{\omega}=0
$$

The velocity field $\mathbf{u}$ can be reconstructed from $\boldsymbol{\omega}$ via the Biot-Savart law:

$$
\mathbf{u}(x)=-\frac{1}{4 \pi} \int_{\mathbb{R}^{3}} \frac{(x-y) \wedge \boldsymbol{\omega}(y)}{|x-y|^{3}} \mathrm{~d} y, \quad x \in \mathbb{R}^{3},
$$

where $\wedge$ denotes the cross product in $\mathbb{R}^{3}$. Although (2) and (9) are formally equivalent, we believe that using the vorticity formulation to compute the long-time asymptotics has a crucial advantage: roughly speaking, the spatial decay of $\boldsymbol{\omega}$ is preserved under the evolution defined by (9). For instance, if $(1+|x|)^{m} \boldsymbol{\omega}_{0} \in L^{2}\left(\mathbb{R}^{3}\right)^{3}$ for some $m \geq 0$, then (9) has a unique local solution $\boldsymbol{\omega}(x, t)$ with initial data $\boldsymbol{\omega}_{0}$ satisfying $(1+|x|)^{m} \boldsymbol{\omega}(\cdot, t) \in$ $L^{2}\left(\mathbb{R}^{3}\right)^{3}$ whenever it exists. Again, we point out that this property does not hold for the velocity field $\mathbf{u}(x, t)$ if $m \geq 5 / 2$. This is the reason why the integrability condition $(1+|x|) \mathbf{u} \in L^{1}\left(\mathbb{R}^{3}\right)^{3}$ is not preserved under evolution.

In the sequel, we always assume that the vorticity $\boldsymbol{\omega}(x, t)$ is small and decreases sufficiently fast as $|x| \rightarrow \infty$. The smallness assumption is not a restriction as far as the long-time behavior is concerned, since all global solutions of the Navier-Stokes equation (in the energy space) converge to zero as $t \rightarrow \infty$. Moreover, this hypothesis allows to deal with global strong solutions of (9). On the other hand, assuming that the vorticity decreases rapidly as $|x| \rightarrow \infty$ is very reasonable from a physical point of view. This is the case, for instance, if the initial data are created by stirring the fluid with a (finite size) tool. In addition, this property is very helpful to study the long-time asymptotics, since in parabolic equations the spatial and temporal behaviors of the solutions are intimately connected.

To actually compute the asymptotics, we express the vorticity $\boldsymbol{\omega}(x, t)$ in terms of the self-similar variables $(\xi, \tau)$ defined by $\xi=x / \sqrt{1+t}, \tau=\log (1+t)$, see (18) below. Although the transformation is time-dependent, the rescaled vorticity $\mathbf{w}(\xi, \tau)$ still satisfies an autonomous equation, as a consequence of the scaling invariance (3). Linearizing this equation around the origin $\mathbf{w}=0$, we find that the generator $\Lambda$ of the time evolution has a countable set of real, isolated eigenvalues with finite multiplicities, and that the essential spectrum can be pushed arbitrarily far away into the left-half plane by choosing the function space (i.e., the spatial decay of the vorticity) appropriately. Thus, the long- 
time asymptotics in a neighborhood of the origin are determined, at any prescribed order, by a finite system of ordinary differential equations.

This reduction procedure, or some variant of it, has been often applied to investigate the long-time behavior of solutions of nonlinear parabolic or damped hyperbolic equations [2], [7], [6], [8], [10], [11], [12], [16], [23], [25]. In the context of the Navier-Stokes equation, rescaling techniques were used by $\mathrm{A}$. Carpio [4], [5] to study the vorticity equations in two and three dimensions. In [3], M. Cannone and F. Planchon constructed a large family of self-similar solutions of the three-dimensional Navier-Stokes equation. These solutions correspond to fixed points of our rescaled vorticity equation, but do not belong to the function spaces we use, because they decay too slowly as $|x| \rightarrow \infty$. In a companion paper [13, we follow the procedure outlined above to study the solutions of the two-dimensional Navier-Stokes and vorticity equations. In addition, we exploit the fact that the spectrum of the generator $\Lambda$ is discrete to construct finite-dimensional invariant manifolds that are approached, at a prescribed rate, by all solutions in a neighborhood of the origin.

The rest of this paper is organized as follows. In Section 2, we prove the existence of global solutions of the vorticity equation (9) in a neighborhood of the origin, and we estimate their decay rate as $t \rightarrow \infty$. The results we obtain are comparable to those of M. Wiegner in [24]. Section 3 is devoted to the first order asymptotics. Under appropriate conditions, we show that

$$
\boldsymbol{\omega}(x, t) \sim \sum_{i=1}^{3} \frac{b_{i}}{(1+t)^{2}} \mathbf{f}_{i}\left(\frac{x}{\sqrt{1+t}}\right), \quad t \rightarrow \infty
$$

where $\mathbf{f}_{1}, \mathbf{f}_{2}, \mathbf{f}_{3}$ are explicit divergence-free vector fields with Gaussian decay at infinity, and $b_{1}, b_{2}, b_{3}$ are real coefficients which can be computed from the initial data. Using (10), a similar result can be obtained for the velocity field $\mathbf{u}(x, t)$. In Section $⿴$, we give a higher order asymptotic expansion of $\boldsymbol{\omega}(x, t)$, including terms of the form $(1+t)^{-5 / 2} \mathbf{g}(x / \sqrt{1+t})$. This result is used in Section 5 to characterize the set of solutions $\boldsymbol{\omega}(x, t)$ of (9) for which the velocity field $\mathbf{u}(x, t)$ satisfies (17). It is shown that these solutions lie on a smooth invariant manifold of finite codimension, which is tangent at the origin to a spectral subspace of the generator $\Lambda$. Intersecting this manifold with the (noninvariant) subspace $\left\{\mathbf{u}_{0} \in L^{2}\left(\mathbb{R}^{3}\right)^{3} \mid(1+|x|) \mathbf{u}_{0} \in L^{1}\left(\mathbb{R}^{3}\right)^{3}\right\}$, we recover exactly conditions (8) in Theorem 1.1. Finally, Appendix $\mathrm{A}$ describes the spectral properties of the generator $\Lambda$, and Appendix B collects various estimates of the velocity field $\mathbf{u}$ in terms of the vorticity $\boldsymbol{\omega}$ in weighted Lebesgue spaces.

Notation. Throughout the paper, we use boldface letters for vector-valued functions, such as $\mathbf{u}(x, t)$ and $\boldsymbol{\omega}(x, t)$. However, to avoid a proliferation of boldface symbols, we use standard italic characters for vector variables, such as $x=\left(x_{1}, x_{2}, x_{3}\right)$. In both cases, $|\cdot|$ denotes the Euclidean norm in $\mathbb{R}^{3}:|\mathbf{u}|=\left(u_{1}^{2}+u_{2}^{2}+u_{3}^{2}\right)^{1 / 2},|x|=\left(x_{1}^{2}+x_{2}^{2}+x_{3}^{2}\right)^{1 / 2}$. For any $p \in[1, \infty]$, we denote by $|f|_{p}$ the norm of a function $f$ in the Lebesgue space $L^{p}\left(\mathbb{R}^{3}\right)$. If $\mathbf{f} \in L^{p}\left(\mathbb{R}^{3}\right)^{3}$, we set $|\mathbf{f}|_{p}=\left.|| \mathbf{f}\right|_{p}$. Weighted norms play a very important role in this paper. We always denote by $\rho: \mathbb{R}^{3} \rightarrow \mathbb{R}$ the weight function defined by $\rho(x)=1+|x|$. For any $m \geq 0$, we set $\|f\|_{m}=\left|\rho^{m} f\right|_{2}$, and $\|\mathbf{f}\|_{m}=\left|\rho^{m} \mathbf{f}\right|_{2}$. If $f \in C^{0}\left([0, T], L^{p}\left(\mathbb{R}^{3}\right)\right)$, we often write $f(\cdot, t)$ or simply $f(t)$ to denote the map $x \mapsto f(x, t)$. Finally, we denote by $C$ a generic positive constant, which may differ from place to place, even in the same chain of inequalities. 
Acknowledgements. Part of this work was done when C.E.W. visited the University of Paris-Sud and Th.G. the Department of Mathematics and Center for BioDynamics of Boston University. The hospitality of both institutions is gratefully acknowledged. We also thank L. Brandolese, I. Gallagher, A. Mielke, G. Raugel, J.-C. Saut, and M. Vishik for stimulating discussions. We are especially indebted to A. Mielke for bringing to our attention the work of [17], which triggered our interest in this problem. The research of C.E.W. is supported in part by the NSF under grant DMS-9803164.

\section{The Cauchy problem for the vorticity equation}

The aim of this section is to prove the existence of global solutions of the vorticity equation for small initial data in weighted Lebesgue spaces. We first recall a few standard estimates for the velocity field $\mathbf{u}$ in terms of the associated vorticity $\boldsymbol{\omega}=\operatorname{rot} \mathbf{u}$. Further estimates in weighted spaces can be found in Appendix B.

Lemma 2.1 Let $\mathbf{u}$ be the velocity field obtained from $\boldsymbol{\omega}$ via the Biot-Savart law (10).

(a) Assume that $1<p<q<\infty$ and $\frac{1}{q}=\frac{1}{p}-\frac{1}{3}$. If $\boldsymbol{\omega} \in L^{p}\left(\mathbb{R}^{3}\right)^{3}$, then $\mathbf{u} \in L^{q}\left(\mathbb{R}^{3}\right)^{3}$, and there exists $C>0$ such that

$$
|\mathbf{u}|_{q} \leq C|\boldsymbol{\omega}|_{p}
$$

(b) Assume that $1 \leq p<3<q \leq \infty$, and define $\alpha \in(0,1)$ by the relation $\frac{1}{3}=\frac{\alpha}{p}+\frac{1-\alpha}{q}$. If $\boldsymbol{\omega} \in L^{p}\left(\mathbb{R}^{3}\right)^{3} \cap L^{q}\left(\mathbb{R}^{3}\right)^{3}$, then $\mathbf{u} \in L^{\infty}\left(\mathbb{R}^{3}\right)^{3}$, and there exists $C>0$ such that

$$
|\mathbf{u}|_{\infty} \leq C|\boldsymbol{\omega}|_{p}^{\alpha}|\boldsymbol{\omega}|_{q}^{1-\alpha}
$$

(c) Assume that $1<p<\infty$. If $\boldsymbol{\omega} \in L^{p}\left(\mathbb{R}^{3}\right)^{3}$, then $\nabla \mathbf{u} \in L^{p}\left(\mathbb{R}^{3}\right)^{9}$ and there exists $C>0$ such that

$$
|\nabla \mathbf{u}|_{p} \leq C|\boldsymbol{\omega}|_{p}
$$

In addition, $\operatorname{div} \mathbf{u}=0$ and, if $\operatorname{div} \boldsymbol{\omega}=0$, then $\operatorname{rot} \mathbf{u}=\boldsymbol{\omega}$.

Proof: Part (a) is a direct consequence of (10) and of the Hardy-Littlewood-Sobolev inequality, see for instance Stein [22], Theorem V.1. To prove (b), assume that $\boldsymbol{\omega} \not \equiv 0$, and let $R=\left(|\omega|_{p} /|\omega|_{q}\right)^{\beta}$, where $\beta=\frac{\alpha}{1-3 / q}=\frac{1-\alpha}{3 / p-1}$. Using Hölder's inequality, we find

$$
\begin{aligned}
|\mathbf{u}(x)| & \leq \frac{1}{4 \pi} \int_{|y| \leq R}|\boldsymbol{\omega}(x-y)| \frac{1}{|y|^{2}} \mathrm{~d} y+\frac{1}{4 \pi} \int_{|y| \geq R}|\boldsymbol{\omega}(x-y)| \frac{1}{|y|^{2}} \mathrm{~d} y \\
& \leq C|\boldsymbol{\omega}|_{q} R^{1-\frac{3}{q}}+C|\boldsymbol{\omega}|_{p} \frac{1}{R^{\frac{3}{p}-1}} \leq 2 C|\boldsymbol{\omega}|_{p}^{\alpha}|\boldsymbol{\omega}|_{q}^{1-\alpha} .
\end{aligned}
$$

Finally, $\nabla \mathbf{u}$ is obtained from $\boldsymbol{\omega}$ via a singular integral kernel of Calderón-Zygmund type, hence (13) follows from Theorem II.3 in 22].

QED

In the sequel, for any $p \in[1, \infty]$, we denote by $\mathrm{L}^{p}\left(\mathbb{R}^{3}\right)$ the function space

$$
\mathrm{L}^{p}\left(\mathbb{R}^{3}\right)=\left\{\mathbf{f} \in L^{p}\left(\mathbb{R}^{3}\right)^{3} \mid \operatorname{div} \mathbf{f}=0\right\},
$$

equipped with the same norm as $L^{p}\left(\mathbb{R}^{3}\right)^{3}$. As is well-known, the $L^{3}$-norm of the velocity field $\mathbf{u}(x, t)$ is invariant under the scaling transformation (3). For the vorticity $\boldsymbol{\omega}(x, t)$, the corresponding critical space is $\mathrm{L}^{3 / 2}\left(\mathbb{R}^{3}\right)$. The following result shows that the Cauchy problem for (9) is globally well-posed for small initial data in $\mathrm{L}^{3 / 2}\left(\mathbb{R}^{3}\right)$. 
Theorem 2.2 There exists $\epsilon_{0}>0$ such that, for all initial data $\boldsymbol{\omega}_{0} \in \mathrm{L}^{3 / 2}\left(\mathbb{R}^{3}\right)$ with $\left|\boldsymbol{\omega}_{0}\right|_{3 / 2} \leq \epsilon_{0}$, (9) has a unique solution $\boldsymbol{\omega} \in C^{0}\left([0, \infty), \mathrm{L}^{3 / 2}\left(\mathbb{R}^{3}\right)\right) \cap C^{0}\left((0, \infty), \mathrm{L}^{\infty}\left(\mathbb{R}^{3}\right)\right)$ satisfying $\boldsymbol{\omega}(0)=\boldsymbol{\omega}_{0}$. Moreover, for all $p \in\left[\frac{3}{2},+\infty\right]$, there exists $C_{p}>0$ such that

$$
|\boldsymbol{\omega}(t)|_{p} \leq \frac{C_{p}\left|\boldsymbol{\omega}_{0}\right|_{3 / 2}}{t^{1-\frac{3}{2 p}}}, \quad t>0 .
$$

Finally, if $\mathbf{u}(x, t)$ is the velocity field obtained from $\boldsymbol{\omega}(x, t)$ via the Biot-Savart law (10), then $\mathbf{u}(\cdot, t) \in \mathrm{L}^{q}\left(\mathbb{R}^{3}\right)$ for all $q \in[3,+\infty]$ if $t>0$, and there exists $C_{q}>0$ such that

$$
|\mathbf{u}(t)|_{q} \leq \frac{C_{q}\left|\boldsymbol{\omega}_{0}\right|_{3 / 2}}{t^{\frac{1}{2}-\frac{3}{2 q}}}, \quad t>0 .
$$

Proof: The proof of Theorem 2.2 follows exactly the argument of Kato [15] which shows that the Navier-Stokes equation has global solutions for small initial data in $\mathrm{L}^{3}\left(\mathbb{R}^{3}\right)$. The same argument also shows that the Cauchy problem for (9) is locally well-posed in $\mathrm{L}^{3 / 2}\left(\mathbb{R}^{3}\right)$, without smallness assumption on the data. More generally, one can prove that (9) has global solutions for small data in the Morrey space $M^{3 / 2}\left(\mathbb{R}^{3}\right)$, see [14].

Following [13], we now introduce the "scaling variables"

$$
\xi=\frac{x}{\sqrt{1+t}}, \quad \tau=\log (1+t) .
$$

If $\boldsymbol{\omega}(x, t)$ is a solution of (9) and if $\mathbf{u}(x, t)$ is the corresponding velocity field, we set

$$
\begin{aligned}
\boldsymbol{\omega}(x, t) & =\frac{1}{1+t} \mathbf{w}\left(\frac{x}{\sqrt{1+t}}, \log (1+t)\right), \\
\mathbf{u}(x, t) & =\frac{1}{\sqrt{1+t}} \mathbf{v}\left(\frac{x}{\sqrt{1+t}}, \log (1+t)\right) .
\end{aligned}
$$

Then the rescaled vorticity $\mathbf{w}(\xi, \tau)$ satisfies the evolution equation

$$
\partial_{\tau} \mathbf{w}=\Lambda \mathbf{w}-(\mathbf{v} \cdot \nabla) \mathbf{w}+(\mathbf{w} \cdot \nabla) \mathbf{v}, \quad \operatorname{div} \mathbf{w}=0,
$$

where $\Lambda$ is the differential operator

$$
\Lambda=\Delta_{\xi}+\frac{1}{2} \xi \cdot \nabla_{\xi}+1, \quad \xi \in \mathbb{R}^{3} .
$$

The rescaled velocity $\mathbf{v}$ is reconstructed from $\mathbf{w}$ via the Biot-Savart law:

$$
\mathbf{v}(\xi)=-\frac{1}{4 \pi} \int_{\mathbb{R}^{3}} \frac{(\xi-\eta) \wedge \mathbf{w}(\eta)}{|\xi-\eta|^{3}} \mathrm{~d} \eta .
$$

As in the two-dimensional case [13], we shall solve the rescaled vorticity equation in weighted $L^{2}$ spaces. For any $m \geq 0$, we define

$$
L^{2}(m)=\left\{f \in L^{2}\left(\mathbb{R}^{3}\right) \mid\|f\|_{m}<\infty\right\},
$$

where

$$
\|f\|_{m}=\left(\int_{\mathbb{R}^{3}}(1+|\xi|)^{2 m}|f(\xi)|^{2} \mathrm{~d} \xi\right)^{1 / 2}=\left|\rho^{m} f\right|_{2} .
$$


Here and in the sequel, we denote by $\rho$ the weight function $\rho(\xi)=1+|\xi|$. In analogy with (14), we introduce the space of divergence free vector fields

$$
\mathrm{L}^{2}(m)=\left\{\mathbf{f} \in L^{2}(m)^{3} \mid \operatorname{div} \mathbf{f}=0\right\},
$$

equipped with the norm $\|\mathbf{f}\|_{m}=\left|\rho^{m}\right| \mathbf{f} \|_{2}$, where $|\mathbf{f}|=\left(f_{1}^{2}+f_{2}^{2}+f_{3}^{2}\right)^{1 / 2}$.

In Appendix A, we show that the operator $\Lambda$ is the generator of a strongly continuous semigroup $\mathrm{e}^{\tau \Lambda}$ in $\mathrm{L}^{2}(m)$, for any $m \geq 0$. Since $\partial_{i} \Lambda=\left(\Lambda+\frac{1}{2}\right) \partial_{i}$ for $i=1,2,3$ (where $\left.\partial_{i}=\partial_{\xi_{i}}\right)$, it is clear that $\partial_{i} \mathrm{e}^{\tau \Lambda}=\mathrm{e}^{\frac{\tau}{2}} \mathrm{e}^{\tau \Lambda} \partial_{i}$ for all $\tau \geq 0$. Thus, using the fact that $\operatorname{div} \mathbf{v}=\operatorname{div} \mathbf{w}=0$, we can rewrite (20) in integral form as follows:

$$
w_{i}(\tau)=\mathrm{e}^{\tau \Lambda} w_{i}(0)+\sum_{j=1}^{3} \int_{0}^{\tau} \partial_{j} \mathrm{e}^{(\tau-s)\left(\Lambda-\frac{1}{2}\right)}\left(w_{j}(s) v_{i}(s)-v_{j}(s) w_{i}(s)\right) \mathrm{d} s,
$$

where $i=1,2,3$. The main result of this section states that, if the initial data are small, (25) has global solutions in $L^{2}(m)$ which decay exponentially to zero as $\tau \rightarrow+\infty$.

Theorem 2.3 Let $0<\mu \leq 1$ and $m>2 \mu+\frac{1}{2}$. There exists $r_{0}>0$ such that, for all initial data $\mathbf{w}_{0} \in \mathrm{L}^{2}(m)$ with $\left\|\mathbf{w}_{0}\right\|_{m} \leq r_{0}$, equation (25) has a unique global solution $\mathbf{w} \in C^{0}\left([0, \infty), \mathrm{L}^{2}(m)\right)$ satisfying $\mathbf{w}(0)=\mathbf{w}_{0}$. In addition, there exists $K_{0} \geq 1$ such that

$$
\|\mathbf{w}(\tau)\|_{m} \leq K_{0} \mathrm{e}^{-\mu \tau}\left\|\mathbf{w}_{0}\right\|_{m}, \quad \tau \geq 0
$$

Proof: Given $\mathbf{w}_{0} \in \mathrm{L}^{2}(m)$, we shall solve (25) in the Banach space

$$
X=\left\{\mathbf{w} \in C^{0}\left([0,+\infty), \mathrm{L}^{2}(m)\right) \mid\|\mathbf{w}\|_{X}=\sup _{\tau \geq 0}\|\mathbf{w}(\tau)\|_{m} \mathrm{e}^{\mu \tau}<\infty\right\} .
$$

We first note that $\tau \mapsto \mathrm{e}^{\tau \Lambda} \mathbf{w}_{0} \in X$; namely, there exists $C_{1} \geq 1$ such that

$$
\left\|\mathrm{e}^{\tau \Lambda} \mathbf{w}_{0}\right\|_{m} \leq C_{1} \mathrm{e}^{-\mu \tau}\left\|\mathbf{w}_{0}\right\|_{m}, \quad \tau \geq 0
$$

Indeed, if $m \leq \frac{5}{2}$, (27) is nothing but (62) with $\epsilon=m-2 \mu-\frac{1}{2}, \alpha=0$, and $n=-1$ or 0 . If $m>\frac{5}{2}$, (27) follows from (63) with $\alpha=0$ and $n=0$.

Next, given $\mathbf{w} \in C^{0}\left([0,+\infty), \mathrm{L}^{2}(m)\right)$, we define $\mathbf{F}[\mathbf{w}] \in C^{0}\left([0,+\infty), \mathrm{L}^{2}(m)\right)$ by

$$
\left(F_{i}[\mathbf{w}]\right)(\tau)=\sum_{j=1}^{3} \int_{0}^{\tau} \partial_{j} \mathrm{e}^{(\tau-s)\left(\Lambda-\frac{1}{2}\right)}\left(w_{j}(s) v_{i}(s)-v_{j}(s) w_{i}(s)\right) \mathrm{d} s, \quad \tau \geq 0 .
$$

We shall prove that $\mathbf{F}$ maps $X$ into $X$, and that there exists $C_{2}>0$ such that

$$
\|\mathbf{F}[\mathbf{w}]\|_{X} \leq C_{2}\|\mathbf{w}\|_{X}^{2}, \quad\|\mathbf{F}[\mathbf{w}]-\mathbf{F}[\tilde{\mathbf{w}}]\|_{X} \leq C_{2}\|\mathbf{w}-\tilde{\mathbf{w}}\|_{X}\left(\|\mathbf{w}\|_{X}+\|\tilde{\mathbf{w}}\|_{X}\right)
$$

for all $\mathbf{w}, \tilde{\mathbf{w}} \in X$. As is easily verified, the bounds (27), (29) imply that the map $\mathbf{w} \mapsto$ $\mathrm{e}^{\tau \Lambda} \mathbf{w}_{0}+\mathbf{F}[\mathbf{w}]$ has a unique fixed point in the ball $\left\{\mathbf{w} \in X \mid\|\mathbf{w}\|_{X} \leq R\right\}$ if $R<\left(2 C_{2}\right)^{-1}$ and $\left\|\mathbf{w}_{0}\right\|_{m} \leq\left(2 C_{1}\right)^{-1} R$. Using Gronwall's lemma, it is then straightforward to show that this fixed point is actually the unique solution of (25) in the space $C^{0}\left([0,+\infty), \mathrm{L}^{2}(m)\right)$. Finally, since $\|\mathbf{w}\|_{X} \leq C_{1}\left\|\mathbf{w}_{0}\right\|_{m}+C_{2}\|\mathbf{w}\|_{X}^{2} \leq C_{1}\left\|\mathbf{w}_{0}\right\|_{m}+\frac{1}{2}\|\mathbf{w}\|_{X}$, the bound (26) holds with $K_{0}=2 C_{1}$. 
To prove (29), we use the following estimate, which is a consequence of Propositions A.3 and A.4. Assume that $f: \mathbb{R}^{3} \rightarrow \mathbb{R}$ satisfies $\rho^{m} f \in L^{3 / 2}\left(\mathbb{R}^{3}\right)$, where $\rho(\xi)=1+|\xi|$. Then there exists $C_{3}>0$ such that, for $j=1,2,3$,

$$
\left\|\partial_{j} \mathrm{e}^{\tau \Lambda} f\right\|_{m} \leq C_{3} \frac{\mathrm{e}^{-\nu \tau}}{a(\tau)^{3 / 4}}\left|\rho^{m} f\right|_{3 / 2}, \quad \tau>0
$$

where $a(\tau)=1-\mathrm{e}^{-\tau}$ and $\nu=\min \left(\mu, \frac{1}{2}\right)$. Indeed, if $0<\tau<2$, then (30) follows from (64) with $p=2$ and $q=3 / 2$. If $\tau \geq 2$, we have

$$
\left\|\partial_{j} \mathrm{e}^{(\tau-1) \Lambda} \mathrm{e}^{\Lambda} f\right\|_{m} \leq C \mathrm{e}^{-\nu(\tau-1)}\left\|\mathrm{e}^{\Lambda} f\right\|_{m} \leq C^{\prime} \mathrm{e}^{-\nu \tau}\left|\rho^{m} f\right|_{3 / 2}
$$

where the second inequality is again a consequence of (64). The first inequality in (31) follows from (62) with $\epsilon=m-2 \mu-\frac{1}{2}$ and $n=-1$ if $m \leq 3 / 2$, and from (63) with $n=-1$ if $m>3 / 2$. This proves (30) for all $\tau>0$.

Given $\mathbf{w} \in X$ and $s \geq 0$, we apply (30) to $f=w_{j}(s) v_{i}(s)-v_{j}(s) w_{i}(s)$, where $i, j \in\{1,2,3\}$ and $\mathbf{v}=\left(v_{1}, v_{2}, v_{3}\right)$ is the velocity field obtained from $\mathbf{w}$ via the BiotSavart law. Using Hölder's inequality and Lemma 2.1, we can bound

$$
\left|\rho^{m} w_{j} v_{i}\right|_{3 / 2} \leq\left|\rho^{m} w_{j}\right|_{2}\left|v_{i}\right|_{6} \leq C\left\|w_{j}\right\|_{m}\left|w_{i}\right|_{2} \leq C\left\|w_{j}\right\|_{m}\left\|w_{i}\right\|_{m},
$$

so that $\left|\rho^{m} f\right|_{3 / 2} \leq C_{4}\|\mathbf{w}(s)\|_{m}^{2}$ for some $C_{4}>0$. Combining this bound with (30) and using (28), we obtain, for all $\tau>0$,

$$
\begin{aligned}
\left\|F_{i}([\mathbf{w}])(\tau)\right\|_{m} & \leq 3 C_{3} C_{4} \int_{0}^{\tau} \frac{\mathrm{e}^{-\left(\nu+\frac{1}{2}\right)(\tau-s)}}{a(\tau-s)^{3 / 4}}\|\mathbf{w}(s)\|_{m}^{2} \mathrm{~d} s \\
& \leq 3 C_{3} C_{4}\|\mathbf{w}\|_{X}^{2} \int_{0}^{\tau} \frac{\mathrm{e}^{-\left(\nu+\frac{1}{2}\right)(\tau-s)}}{a(\tau-s)^{3 / 4}} \mathrm{e}^{-2 \mu s} \mathrm{~d} s \leq C_{2}\|\mathbf{w}\|_{X}^{2} \mathrm{e}^{-\mu \tau}
\end{aligned}
$$

since $\nu+\frac{1}{2} \geq \mu$. This establishes the first inequality in (29), and the second one can be proved along the same lines. The proof of Theorem 2.3 is now complete.

QED

Since the semigroup $\mathrm{e}^{\tau \Lambda}$ is not analytic in $\mathrm{L}^{2}(m)$, the solution $\mathbf{w}$ given by Theorem 2.3 is in general not a smooth function of $\tau$. In particular, $\tau \mapsto \mathbf{w}(\tau) \notin C^{1}\left((0,+\infty), \mathrm{L}^{2}(m)\right)$, so that $\mathbf{w}$ is not a classical solution of (20) in $\mathrm{L}^{2}(m)$. Nevertheless, following the common use, we shall often refer to $\mathbf{w}$ as to the (mild) solution of $(20)$ in $\mathrm{L}^{2}(m)$. Remark that the evolution defined by $(20)$ is regularizing in the sense that $\mathbf{w}(\xi, \tau)$ is a smooth function of $\xi \in \mathbb{R}^{3}$ for any $\tau>0$. This property is well-known, and will not be proved here. We only quote the following result:

Proposition 2.4 Let $0<\mu \leq 1, m>2 \mu+\frac{1}{2}$, and let $\mathbf{w} \in C^{0}\left([0, \infty), \mathrm{L}^{2}(m)\right)$ be the solution of (20) given by Theorem 2.3. There exists $K_{1}>0$ such that, for all $p \in[2,+\infty]$,

$$
\left|\rho^{m} \mathbf{w}(\tau)\right|_{p} \leq K_{1}\left(1+\tau^{-\gamma_{p}}\right) \mathrm{e}^{-\mu \tau}\left\|\mathbf{w}_{0}\right\|_{m}, \quad \tau>0
$$

where $\rho(\xi)=1+|\xi|$ and $\gamma_{p}=\frac{3}{2}\left(\frac{1}{2}-\frac{1}{p}\right)$.

Proof: In view of (26), it is clearly sufficient to prove (33) for $0<\tau \leq 1$. This can be done by a standard bootstrap argument, using Proposition A.4, Lemma 2.1, and the integral equation (25) satisfied by $\mathbf{w}$. We omit the details.

QED 
Corollary 2.5 Let $0<\mu \leq 1$ and $m>2 \mu+\frac{1}{2}$. Let $\mathbf{w} \in C^{0}\left([0, \infty), \mathrm{L}^{2}(m)\right)$ be the solution of (20) given by Theorem [2.5, and let $\mathbf{v}$ be the corresponding velocity field. There exists $K_{2}>0$ such that, for all $\tau \geq 1$,

$$
|\mathbf{w}(\tau)|_{p} \leq K_{2} \mathrm{e}^{-\mu \tau}\left\|\mathbf{w}_{0}\right\|_{m} \quad \text { for all } \begin{cases}p \in\left(p_{m}, \infty\right] & \text { if } \frac{1}{2}<m \leq \frac{3}{2} \\ p \in[1, \infty] & \text { if } m>\frac{3}{2}\end{cases}
$$

and

$$
|\mathbf{v}(\tau)|_{q} \leq K_{2} \mathrm{e}^{-\mu \tau}\left\|\mathbf{w}_{0}\right\|_{m} \quad \text { for all } \begin{cases}q \in\left(q_{m}, \infty\right] & \text { if } \frac{1}{2}<m \leq \frac{5}{2}, \\ q \in(1, \infty] & \text { if } m>\frac{5}{2},\end{cases}
$$

where $p_{m}=\frac{6}{3+2 m}$ and $q_{m}=\frac{6}{1+2 m}$. Moreover, the bounds (34), (35) hold for all $\tau \geq 0$ if $p \leq 2, q \leq 6$.

Proof: If $p>2$, (34) is a direct consequence of (33). If $p_{m}<p \leq 2$, then $|\mathbf{w}|_{p} \leq C\left|\rho^{m} \mathbf{w}\right|_{2}$ by Hölder's inequality, and (34) again follows from (33). Using (34) and Lemma 2.1, we easily obtain (35) if $q>3 / 2$. Finally, if $m>3 / 2$ and $\max \left(1, q_{m}\right)<q \leq 3 / 2$, we can pick $n<\min (m, 5 / 2)$ such that $q>q_{n}$. Using Hölder's inequality and Proposition B.1, we find $|\mathbf{v}|_{q} \leq C\left|\rho^{n} \mathbf{v}\right|_{6} \leq C\left|\rho^{n} \mathbf{w}\right|_{2} \leq C\|\mathbf{w}\|_{m}$, and (35) follows.

QED

Remark 2.6 The fact that the value $q=1$ is excluded in (35) if $m>\frac{5}{2}$ is not a technical restriction. As is shown in Corollary B.4, the velocity field $\mathbf{v}(\xi, \tau)$ is not integrable in this case, unless $\int_{\mathbb{R}^{3}} \xi_{i} w_{j}(\xi, \tau) \mathrm{d} \xi=0$ for all $i, j \in\{1,2,3\}$.

For the vorticity $\boldsymbol{\omega}(x, t)$ and the velocity $\mathbf{u}(x, t)$ in the original variables, Corollary 2.5 implies, for the same values of $p$ and $q$ :

$$
|\boldsymbol{\omega}(t)|_{p} \leq K_{2} t^{-1-\mu+\frac{3}{2 p}}\left\|\boldsymbol{\omega}_{0}\right\|_{m}, \quad|\mathbf{u}(t)|_{q} \leq K_{2} t^{-\frac{1}{2}-\mu+\frac{3}{2 q}}\left\|\boldsymbol{\omega}_{0}\right\|_{m}, \quad t \geq 1 .
$$

We now explain our motivation for introducing the scaling variables (17). As is shown in Appendix A, the spectrum of $\Lambda$ acting on $\mathrm{L}^{2}(m)$ can be decomposed as $\sigma(\Lambda)=\sigma_{d}(\Lambda) \cup$ $\sigma_{c}(\Lambda)$, where

$$
\sigma_{d}(\Lambda)=\left\{-\frac{k+1}{2} \mid k \in \mathbb{N}^{*}\right\}, \quad \sigma_{c}(\Lambda)=\left\{\lambda \in \mathbb{C} \mid \Re(\lambda) \leq \frac{1}{4}-\frac{m}{2}\right\} .
$$

(See Fig. 1.) Remark that the discrete spectrum $\sigma_{d}(\Lambda)$ does not depend on $m$, whereas the continuous spectrum $\sigma_{c}(\Lambda)$ can be shifted arbitrarily far away from the origin by choosing $m$ appropriately. Therefore, if $m \geq 0$ is sufficiently large, the long-time behavior of the solutions of (20) in a neighborhood of the origin is governed by a finite system of ordinary differential equations. This system is obtained by projecting (20) onto the finite-dimensional subspace of $\mathrm{L}^{2}(m)$ spanned by the eigenfunctions of $\Lambda$ corresponding to the first eigenvalues $\lambda_{k}=-\frac{k+1}{2}$, with $k=1,2, \ldots, k_{0}$. 


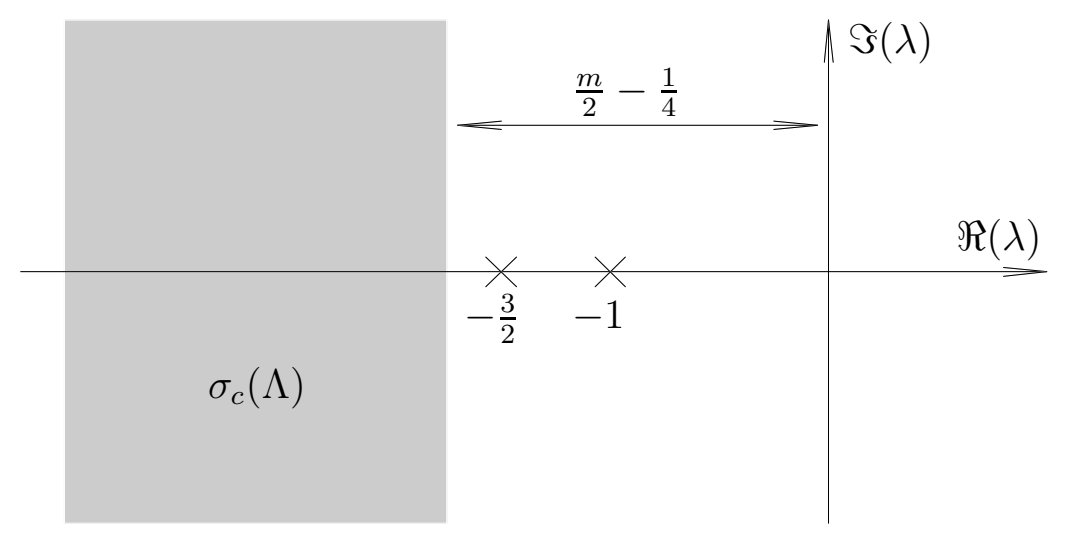

Fig. 1: The spectrum of the linear operator $\Lambda$ in $\mathrm{L}^{2}(m)$, when $m=4$.

A rigorous justification of this reduction, using invariant manifold theory, can be found in [13] for the two-dimensional vorticity equation. Specifically, given any $\nu>0$, we prove the existence of a finite-dimensional locally invariant manifold, which is tangent at the origin to the spectral subspace corresponding to the first eigenvalues of $\Lambda$, and which is approached at a rate $\mathcal{O}\left(\mathrm{e}^{-\nu \tau}\right)$ or faster by any solution of (20) that stays in a neighborhood of the origin for all times. This method allows, at least in principle, to compute the longtime asymptotics of the solutions to arbitrarily high order by studying a finite-dimensional dynamical system - the restriction of the rescaled vorticity equation to the manifold.

Invariant manifolds can be constructed in the three-dimensional case also, and we use them in our discussion of the set of solutions of the Navier-Stokes equations which decay "faster than expected" in Section 5. However, for computing the asymptotics, we show that one can also use a different approach. Given $k_{0} \in \mathbb{N}^{*}$ and $m>k_{0}+\frac{3}{2}$, we decompose any solution $\mathbf{w}$ of (20) in $\mathrm{L}^{2}(m)$ as

$$
\mathbf{w}(\xi, \tau)=\sum_{k=1}^{k_{0}} \sum_{\ell=1}^{k(k+2)} \alpha_{k \ell}(\tau) \mathbf{w}_{k \ell}(\xi)+\mathbf{R}(\xi, \tau),
$$

where $\alpha_{k \ell} \in \mathbb{R}$ and, for any $k \in\left\{1, \ldots, k_{0}\right\},\left\{\mathbf{w}_{k \ell} \mid \ell=1, \ldots, k(k+2)\right\}$ is a basis of the eigenspace $\left\{\mathbf{w} \in \mathrm{L}^{2}(m) \mid \Lambda \mathbf{w}=-\frac{k+1}{2} \mathbf{w}\right\}$. Using this decomposition, (20) becomes a system of ordinary differential equations for the coefficients $\alpha_{k \ell}$ coupled to a partial differential equation for the remainder $\mathbf{R}$. A direct analysis of this system allows to compute the asymptotics up to order $\mathcal{O}\left(\mathrm{e}^{-\nu \tau}\right)$, where $\nu=\min \left(\frac{k_{0}+2}{2}, \frac{m}{2}-\frac{1}{4}\right)$. This program is carried out in Section 3 for $k_{0}=1$ (first order asymptotics) and in Section 6 for $k_{0}=2$ (second order asymptotics).

\section{$3 \quad$ First-order asymptotics}

In this section, we consider the behavior of the solutions of (20) in $\mathrm{L}^{2}(m)$ with $\frac{5}{2}<m \leq \frac{7}{2}$. In this space, the discrete spectrum of $\Lambda$ consists of a single isolated eigenvalue $\lambda_{1}=-1$, of multiplicity 3. A convenient basis of eigenvectors is given by $\left\{\mathbf{f}_{1}, \mathbf{f}_{2}, \mathbf{f}_{3}\right\}$, where $\mathbf{f}_{i}=$ 
$\operatorname{rot}\left(G \mathbf{e}_{i}\right)$. Here and in the sequel, $G$ is the Gaussian function

$$
G(\xi)=\frac{1}{(4 \pi)^{3 / 2}} \mathrm{e}^{-|\xi|^{2} / 4}, \quad \xi \in \mathbb{R}^{3}
$$

and $\left\{\mathbf{e}_{1}, \mathbf{e}_{2}, \mathbf{e}_{3}\right\}$ denotes the canonical basis of $\mathbb{R}^{3}$. A short calculation shows that $\mathbf{f}_{i}=\mathbf{p}_{i} G$ for $i=1,2,3$, where $\mathbf{p}_{i}(\xi)=\frac{1}{2}\left(\mathbf{e}_{i} \wedge \xi\right)$. Explicitly,

$$
\mathbf{p}_{1}(\xi)=\frac{1}{2}\left(\begin{array}{c}
0 \\
-\xi_{3} \\
\xi_{2}
\end{array}\right), \quad \mathbf{p}_{2}(\xi)=\frac{1}{2}\left(\begin{array}{c}
\xi_{3} \\
0 \\
-\xi_{1}
\end{array}\right), \quad \mathbf{p}_{3}(\xi)=\frac{1}{2}\left(\begin{array}{c}
-\xi_{2} \\
\xi_{1} \\
0
\end{array}\right)
$$

The vector fields $\mathbf{p}_{i}$ satisfy $\operatorname{div} \mathbf{p}_{i}=0$ and $\operatorname{rot} \mathbf{p}_{i}=\mathbf{e}_{i}$. Integrating by parts, we thus find

$$
\int_{\mathbb{R}^{3}} \mathbf{p}_{i} \cdot \mathbf{f}_{j} \mathrm{~d} \xi=\int_{\mathbb{R}^{3}} \operatorname{rot}\left(\mathbf{p}_{i}\right) \cdot\left(G \mathbf{e}_{j}\right) \mathrm{d} \xi=\left(\mathbf{e}_{i} \cdot \mathbf{e}_{j}\right) \int_{\mathbb{R}^{3}} G \mathrm{~d} \xi=\delta_{i j} .
$$

Moreover, if $\Lambda^{*}=\Delta-\frac{1}{2}(\xi \cdot \nabla)-\frac{1}{2}$ is the formal adjoint of $\Lambda$, it is easy to verify that $\Lambda^{*} \mathbf{p}_{i}=-\mathbf{p}_{i}$ for $i=1,2,3$. The velocity fields $\mathbf{v}^{\mathbf{f}_{i}}$ corresponding to $\mathbf{f}_{i}$ are computed in Appendix B. In particular, we mention that $\left|\mathbf{v}^{\mathbf{f}_{i}}(\xi)\right| \sim|\xi|^{-3}$ as $|\xi| \rightarrow \infty$, so that $\mathbf{v}^{\mathbf{f}_{i}} \in \mathrm{L}^{q}\left(\mathbb{R}^{3}\right)$ for all $q>1$. Using these notations, any solution $\mathbf{w}$ of $(20)$ in $\mathrm{L}^{2}(m)$ can be decomposed as

$$
\mathbf{w}(\xi, \tau)=\sum_{i=1}^{3} \beta_{i}(\tau) \mathbf{f}_{i}(\xi)+\mathbf{R}(\xi, \tau)
$$

where

$$
\beta_{i}(\tau)=\int_{\mathbb{R}^{3}} \mathbf{p}_{i}(\xi) \cdot \mathbf{w}(\xi, \tau) \mathrm{d} \xi, \quad i=1,2,3 .
$$

Then $\mathbf{R}(\cdot, \tau)$ belongs to the subspace $\mathcal{W}_{1}$ of $\mathrm{L}^{2}(m)$ defined in $(61)$, which is also the spectral subspace associated with the continuous spectrum $\left\{\lambda \in \mathbb{C} \mid \Re(\lambda) \leq \frac{1}{4}-\frac{m}{2}\right\}$ of the operator $\Lambda$. As in the two-dimensional case [13], the coefficients $\beta_{i}$ obey a linear evolution equation:

Lemma 3.1 Assume that $m>\frac{5}{2}$, and let $\mathbf{w} \in C^{0}\left([0, T], \mathrm{L}^{2}(m)\right)$ be a solution of (20). Then the coefficients $\beta_{i}$ defined by (38) satisfy, for all $\tau \in[0, T]$,

$$
\dot{\beta}_{i}(\tau)=-\beta_{i}(\tau), \quad i=1,2,3 .
$$

Proof: Since $\operatorname{rot}(\mathbf{v} \wedge \mathbf{w})=(\mathbf{w} \cdot \nabla) \mathbf{v}-(\mathbf{v} \cdot \nabla) \mathbf{w},(20)$ is equivalent to

$$
\partial_{\tau} \mathbf{w}=\Lambda \mathbf{w}+\operatorname{rot}(\mathbf{v} \wedge \mathbf{w}), \quad \operatorname{div} \mathbf{w}=0 .
$$

Differentiating (38) formally with respect to $\tau$ and integrating by parts, we thus find

$$
\dot{\beta}_{i}=\int_{\mathbb{R}^{3}} \mathbf{p}_{i} \cdot(\Lambda \mathbf{w}+\operatorname{rot}(\mathbf{v} \wedge \mathbf{w})) \mathrm{d} \xi=-\beta_{i}+\int_{\mathbb{R}^{3}} \mathbf{e}_{i} \cdot(\mathbf{v} \wedge \mathbf{w}) \mathrm{d} \xi
$$

where we used the fact that $\Lambda^{*} \mathbf{p}_{i}=-\mathbf{p}_{i}$. Since the right-hand side of (39) belongs to $C^{0}([0, T])$ and depends continuously on $\mathbf{w}$, the calculations above can be justified by a density argument. In particular, $\beta_{i} \in C^{1}([0, T])$ for $i=1,2,3$. Finally, using the identity 
$\mathbf{v} \wedge \mathbf{w}=\mathbf{v} \wedge \operatorname{rot} \mathbf{v}=\frac{1}{2} \nabla|\mathbf{v}|^{2}-(\mathbf{v} \cdot \nabla) \mathbf{v}$ and the fact that $\operatorname{div} \mathbf{v}=0$, we see that the last integral in (39) vanishes, hence $\dot{\beta}_{i}=-\beta_{i}$.

QED

In particular, it follows from Lemma 3.1 that the subspace $\mathcal{W}_{1}$ is invariant under the evolution defined by (20). The remainder $\mathbf{R}$ in (37) satisfies the equation

$$
\partial_{\tau} \mathbf{R}=\Lambda \mathbf{R}+Q_{1}((\mathbf{w} \cdot \nabla) \mathbf{v}-(\mathbf{v} \cdot \nabla) \mathbf{w}), \quad \operatorname{div} \mathbf{R}=0
$$

where $Q_{1}: \mathrm{L}^{2}(m) \rightarrow \mathcal{W}_{1}$ is the spectral projection (for the operator $\Lambda$ ) onto the subspace $\mathcal{W}_{1}$. Explicitly,

$$
Q_{1} \mathbf{w}=\mathbf{w}-\sum_{i=1}^{3}\left(\int_{\mathbb{R}^{3}} \mathbf{p}_{i} \cdot \mathbf{w} \mathrm{d} \xi\right) \mathbf{f}_{i} .
$$

The following result describes the first order asymptotics of $\mathbf{w}(\tau)$ as $\tau \rightarrow \infty$.

Theorem 3.2 Let $1<\nu \leq \frac{3}{2}, m>2 \nu+\frac{1}{2}$, and let $\mathbf{w} \in C^{0}\left([0, \infty), \mathrm{L}^{2}(m)\right)$ be the solution of (20) given by Theorem 2.3 with $\mu=1$. Then there exists $K_{3} \geq 1$ such that

$$
\left\|\mathbf{w}(\tau)-\sum_{i=1}^{3} b_{i} \mathrm{e}^{-\tau} \mathbf{f}_{i}\right\|_{m} \leq K_{3} \mathrm{e}^{-\nu \tau}\left\|\mathbf{w}_{0}\right\|_{m}, \quad \tau \geq 0,
$$

where $b_{i}=\int_{\mathbb{R}^{3}} \mathbf{p}_{i} \cdot \mathbf{w}_{0} \mathrm{~d} \xi, i=1,2,3$.

Proof: If $\mathbf{w} \in C^{0}\left([0, \infty), \mathrm{L}^{2}(m)\right)$ is the solution of (20) given by Theorem 2.3 and $\mathbf{v}$ the corresponding velocity field, we define $\beta_{i}$ and $\mathbf{R}$ by (37), (38). By Lemma 3.1, $\beta_{i}(\tau)=$ $b_{i} \mathrm{e}^{-\tau}$ for $i=1,2,3$. To bound the remainder $\mathbf{R}$, we use the integral equation

$$
\mathbf{R}(\tau)=\mathrm{e}^{\tau \Lambda} \mathbf{R}_{0}+\int_{0}^{\tau} Q_{1} \boldsymbol{\Phi}(\tau-s, \mathbf{w}(s), \mathbf{v}(s)) \mathrm{d} s
$$

where $\mathbf{R}_{0}=Q_{1} \mathbf{w}_{0}$ and $\boldsymbol{\Phi}(\sigma, \mathbf{w}, \mathbf{v})=\mathrm{e}^{\sigma \Lambda}((\mathbf{w} \cdot \nabla) \mathbf{v}-(\mathbf{v} \cdot \nabla) \mathbf{w})$.

By Proposition A.3, there exists $C_{1}>0$ such that

$$
\left\|\mathrm{e}^{\tau \Lambda} \mathbf{R}_{0}\right\|_{m} \leq C_{1} \mathrm{e}^{-\nu \tau}\left\|\mathbf{w}_{0}\right\|_{m}, \quad \tau \geq 0
$$

Indeed, if $\frac{5}{2}<m \leq \frac{7}{2}$, (41) is a consequence of (62) with $\alpha=0, n=1$, and $\epsilon=m-2 \nu-\frac{1}{2}$. If $m>\frac{7}{2}$, (41) follows from (63) with $\alpha=0$ and $n=1$.

To estimate the integral in (40), we proceed as in the proof of Theorem 2.3. Exchanging $\nabla$ with $\mathrm{e}^{\sigma \Lambda}$, we can write $\boldsymbol{\Phi}=\left(\Phi_{1}, \Phi_{2}, \Phi_{3}\right)$, where

$$
\Phi_{i}(\sigma, \mathbf{w}, \mathbf{v})=\sum_{j=1}^{3} \partial_{j} \mathrm{e}^{\sigma\left(\Lambda-\frac{1}{2}\right)}\left(w_{j} v_{i}-v_{j} w_{i}\right), \quad i=1,2,3
$$

see (25). By (30), (32), there exists $C_{2}>0$ such that $\left\|Q_{1} \mathbf{\Phi}(\sigma, \mathbf{w}, \mathbf{v})\right\|_{m} \leq C_{2} \sigma^{-3 / 4}\|\mathbf{w}\|_{m}^{2}$ for all $\sigma \in[0,1]$. Using (41) and (26), we thus find

$$
\|\mathbf{R}(\tau)\|_{m} \leq C_{1}\left\|\mathbf{w}_{0}\right\|_{m}+\int_{0}^{\tau} C_{2}(\tau-s)^{-3 / 4}\left(K_{0}\left\|\mathbf{w}_{0}\right\|_{m}\right)^{2} \mathrm{~d} s \leq C\left\|\mathbf{w}_{0}\right\|_{m},
$$


for all $\tau \in[0,1]$. (Here and in the sequel, we use the fact that $\left\|\mathbf{w}_{0}\right\|_{m} \leq r_{0}$, see Theorem 2.3.) If $\tau>1$, we write $\mathbf{R}(\tau)=\mathrm{e}^{\tau \Lambda} \mathbf{R}_{0}+\mathbf{R}_{1}(\tau)+\mathbf{R}_{2}(\tau)$, where

$$
\begin{aligned}
& \mathbf{R}_{1}(\tau)=\int_{0}^{\tau-1} \mathrm{e}^{(\tau-1-s) \Lambda} Q_{1} \boldsymbol{\Phi}(1, \mathbf{w}(s), \mathbf{v}(s)) \mathrm{d} s \\
& \mathbf{R}_{2}(\tau)=\int_{\tau-1}^{\tau} Q_{1} \boldsymbol{\Phi}(\tau-s, \mathbf{w}(s), \mathbf{v}(s)) \mathrm{d} s
\end{aligned}
$$

Proceeding as above, we obtain

$$
\begin{aligned}
\left\|\mathbf{R}_{1}(\tau)\right\|_{m} & \leq \int_{0}^{\tau-1} C_{1} \mathrm{e}^{-\nu(\tau-1-s)} C_{2}\left(K_{0} \mathrm{e}^{-s}\left\|\mathbf{w}_{0}\right\|_{m}\right)^{2} \mathrm{~d} s \leq C \mathrm{e}^{-\nu \tau}\left\|\mathbf{w}_{0}\right\|_{m}^{2}, \\
\left\|\mathbf{R}_{2}(\tau)\right\|_{m} & \leq \int_{\tau-1}^{\tau} C_{2}(\tau-s)^{-3 / 4}\left(K_{0} \mathrm{e}^{-s}\left\|\mathbf{w}_{0}\right\|_{m}\right)^{2} \mathrm{~d} s \leq C \mathrm{e}^{-2 \tau}\left\|\mathbf{w}_{0}\right\|_{m}^{2} .
\end{aligned}
$$

Thus, there exists $K_{3}>0$ such that $\|\mathbf{R}(\tau)\|_{m} \leq K_{3} \mathrm{e}^{-\nu \tau}\left\|\mathbf{w}_{0}\right\|_{m}$, for all $\tau \geq 0$.

Corollary 3.3 Let $1<\nu \leq \frac{3}{2}$ and $m>2 \nu+\frac{1}{2}$. Let $\mathbf{w} \in C^{0}\left([0, \infty), \mathrm{L}^{2}(m)\right)$ be the solution of (20) given by Theorem 2.3 with $\mu=1$, and let $\mathbf{v}$ be the corresponding velocity field. There exists $K_{4}>0$ such that, for all $\tau \geq 1$,

$$
\begin{aligned}
& \left|\mathbf{w}(\tau)-\sum_{i=1}^{3} b_{i} \mathrm{e}^{-\tau} \mathbf{f}_{i}\right|_{p} \leq K_{4} \mathrm{e}^{-\nu \tau}\left\|\mathbf{w}_{0}\right\|_{m}, \quad 1 \leq p \leq \infty, \\
& \left|\mathbf{v}(\tau)-\sum_{i=1}^{3} b_{i} \mathrm{e}^{-\tau} \mathbf{v}^{\mathbf{f}_{i}}\right|_{q} \leq K_{4} \mathrm{e}^{-\nu \tau}\left\|\mathbf{w}_{0}\right\|_{m}, \quad 1 \leq q \leq \infty,
\end{aligned}
$$

where $\mathbf{v}^{\mathbf{f}_{i}}$ is given by (68). Moreover, the bounds (49), (43) hold for all $\tau \geq 0$ if $p \leq 2$, $q \leq 6$.

Proof: Using the analogue of Proposition 2.4 for $\mathbf{R}(\xi, \tau)$ and proceeding as in the proof of Corollary 2.5, we obtain (42) for $1 \leq p \leq \infty$ and (43) for $1<q \leq \infty$. To prove (43) for $q=1$, we pick $n \in \mathbb{R}$ such that $5 / 2<n<\min (m, 7 / 2)$. If $\mathbf{v}^{\mathbf{R}}$ is the velocity field obtained from $\mathbf{R}$ via the Biot-Savart law (22), then using Hölder's inequality and Proposition B.1 we can bound $\left|\mathbf{v}^{\mathbf{R}}\right|_{1} \leq C\left|\rho^{n} \mathbf{v}^{\mathbf{R}}\right|_{6} \leq C\left|\rho^{n} \mathbf{R}\right|_{2} \leq C\|\mathbf{R}\|_{m}$, and the result follows. QED

In terms of the original variables, Corollary 3.3 shows that, for all $t \geq 1$,

$$
\begin{aligned}
& \left|\boldsymbol{\omega}(t)-\boldsymbol{\omega}_{a p p}(t)\right|_{p} \leq C t^{-1-\nu+\frac{3}{2 p}}\left\|\boldsymbol{\omega}_{0}\right\|_{m}, \quad 1 \leq p \leq \infty \\
& \left|\mathbf{u}(t)-\mathbf{u}_{\text {app }}(t)\right|_{q} \leq C t^{-\frac{1}{2}-\nu+\frac{3}{2 q}}\left\|\boldsymbol{\omega}_{0}\right\|_{m}, \quad 1 \leq q \leq \infty
\end{aligned}
$$

where $\boldsymbol{\omega}_{a p p}(x, t), \mathbf{u}_{a p p}(x, t)$ are the self-similar vector fields defined by

$$
\boldsymbol{\omega}_{a p p}(x, t)=\sum_{i=1}^{3} \frac{b_{i}}{(1+t)^{2}} \mathbf{f}_{i}\left(\frac{x}{\sqrt{1+t}}\right), \quad \mathbf{u}_{a p p}(x, t)=\sum_{i=1}^{3} \frac{b_{i}}{(1+t)^{3 / 2}} \mathbf{v}^{\mathbf{f}_{i}}\left(\frac{x}{\sqrt{1+t}}\right)
$$

Remark that $\mathbf{u}_{a p p}(\cdot, t) \notin \mathrm{L}^{1}\left(\mathbb{R}^{3}\right)$, unless $b_{1}=b_{2}=b_{3}=0$. 


\section{Second-order asymptotics}

We now turn our attention to the solutions of (20) in $\mathrm{L}^{2}(m)$ with $\frac{7}{2}<m \leq \frac{9}{2}$. Acting on this space, the operator $\Lambda$ has exactly two isolated eigenvalues: $\lambda_{1}=-1$ (of multiplicity 3) and $\lambda_{2}=-\frac{3}{2}$ (of multiplicity 8). Let $\mathcal{E}_{2}$ be the subspace of $\mathrm{L}^{2}(m)$ spanned by the eigenfunctions corresponding to $\lambda_{2}$, see Appendix A. A convenient basis of $\mathcal{E}_{2}$ is provided by the vector fields $\mathbf{g}_{i}$ and $\mathbf{h}_{i j}$, which we now define.

a) For $i=1,2,3$, let $\mathbf{g}_{i}=\operatorname{rot} \mathbf{f}_{i}=\operatorname{rot}\left(\mathbf{p}_{i} G\right)=\frac{G}{4}\left(\left(4-|\xi|^{2}\right) \mathbf{e}_{i}+\xi_{i} \xi\right)$. Explicitly,

$$
\mathbf{g}_{1}=\frac{G}{4}\left(\begin{array}{c}
4-\xi_{2}^{2}-\xi_{3}^{3} \\
\xi_{1} \xi_{2} \\
\xi_{1} \xi_{3}
\end{array}\right), \quad \mathbf{g}_{2}=\frac{G}{4}\left(\begin{array}{c}
\xi_{1} \xi_{2} \\
4-\xi_{1}^{2}-\xi_{3}^{2} \\
\xi_{2} \xi_{3}
\end{array}\right) \quad, \quad \mathbf{g}_{3}=\frac{G}{4}\left(\begin{array}{c}
\xi_{1} \xi_{3} \\
\xi_{2} \xi_{3} \\
4-\xi_{1}^{2}-\xi_{2}^{2}
\end{array}\right)
$$

Then $\operatorname{div} \mathbf{g}_{i}=0$ and $\Lambda \mathbf{g}_{i}=-\frac{3}{2} \mathbf{g}_{i}$. By construction, the velocity field associated to $\mathbf{g}_{i}$ is $\mathbf{v}^{\mathbf{g}_{i}} \equiv \mathbf{f}_{i}$. In particular, $\mathbf{v}^{\mathbf{g}_{i}}$ has a Gaussian decay as $|\xi| \rightarrow \infty$. We also define

$$
\mathbf{q}_{1}(\xi)=\frac{1}{2}\left(\begin{array}{c}
2-\xi_{1}^{2} \\
\xi_{1} \xi_{2} \\
\xi_{1} \xi_{3}
\end{array}\right), \quad \mathbf{q}_{2}(\xi)=\frac{1}{2}\left(\begin{array}{c}
\xi_{1} \xi_{2} \\
2-\xi_{2}^{2} \\
\xi_{2} \xi_{3}
\end{array}\right), \quad \mathbf{q}_{3}(\xi)=\frac{1}{2}\left(\begin{array}{c}
\xi_{1} \xi_{3} \\
\xi_{2} \xi_{3} \\
2-\xi_{3}^{2}
\end{array}\right)
$$

Then $\operatorname{div} \mathbf{q}_{i}=0, \operatorname{rot} \mathbf{q}_{i}=\mathbf{p}_{i}$, and $\Lambda^{*} \mathbf{q}_{i}=-\frac{3}{2} \mathbf{q}_{i}$. It follows that

$$
\int_{\mathbb{R}^{3}} \mathbf{q}_{i} \cdot \mathbf{g}_{j} \mathrm{~d} \xi=\int_{\mathbb{R}^{3}} \operatorname{rot}\left(\mathbf{q}_{i}\right) \cdot \mathbf{f}_{j} \mathrm{~d} \xi=\int_{\mathbb{R}^{3}} \mathbf{p}_{i} \cdot \mathbf{f}_{j} \mathrm{~d} \xi=\delta_{i j} .
$$

b) For $(i j) \in S=\{(11),(12),(13),(22),(23)\}$, we define $\mathbf{h}_{i j}=\partial_{i} \mathbf{f}_{j}+\partial_{j} \mathbf{f}_{i}$. Explicitly, we have $\mathbf{h}_{i i}=-\xi_{i} \mathbf{f}_{i}$ for $i=1,2$ and

$$
\mathbf{h}_{12}=\frac{G}{4}\left(\begin{array}{c}
-\xi_{1} \xi_{3} \\
\xi_{2} \xi_{3} \\
\xi_{1}^{2}-\xi_{2}^{2}
\end{array}\right), \quad \mathbf{h}_{13}=\frac{G}{4}\left(\begin{array}{c}
\xi_{1} \xi_{2} \\
\xi_{3}^{2}-\xi_{1}^{2} \\
-\xi_{2} \xi_{3}
\end{array}\right) \quad, \quad \mathbf{h}_{23}=\frac{G}{4}\left(\begin{array}{c}
\xi_{2}^{2}-\xi_{3}^{2} \\
-\xi_{1} \xi_{2} \\
\xi_{1} \xi_{3}
\end{array}\right)
$$

Then $\operatorname{div} \mathbf{h}_{i j}=0$ and $\Lambda \mathbf{h}_{i j}=-\frac{3}{2} \mathbf{h}_{i j}$ for all $(i j) \in S$. The velocity fields $\mathbf{v}^{\mathbf{h}_{i j}}$ corresponding to $\mathbf{h}_{i j}$ are computed in Appendix B. In particular, we remark that $\left|\mathbf{v}^{\mathbf{h}_{i j}}(\xi)\right| \sim|\xi|^{-4}$ as $|\xi| \rightarrow \infty$, so that $\rho \mathbf{v}^{\mathbf{h}_{i j}} \notin L^{1}\left(\mathbb{R}^{3}\right)^{3}$. We also define $\mathbf{r}_{11}=\frac{1}{2} \xi_{1} \xi_{3} \mathbf{e}_{2}, \mathbf{r}_{22}=-\frac{1}{2} \xi_{2} \xi_{3} \mathbf{e}_{1}$, and

$$
\mathbf{r}_{12}=\frac{1}{2}\left(\begin{array}{c}
-\xi_{1} \xi_{3} \\
\xi_{2} \xi_{3} \\
0
\end{array}\right), \quad \mathbf{r}_{13}=\frac{1}{2}\left(\begin{array}{c}
\xi_{1} \xi_{2} \\
0 \\
-\xi_{2} \xi_{3}
\end{array}\right), \quad \mathbf{r}_{23}=\frac{1}{2}\left(\begin{array}{c}
0 \\
-\xi_{1} \xi_{2} \\
\xi_{1} \xi_{3}
\end{array}\right)
$$

Then $\operatorname{div} \mathbf{r}_{i j}=0$ and $\Lambda^{*} \mathbf{r}_{i j}=-\frac{3}{2} \mathbf{r}_{i j}$ for all $(i j) \in S$. A direct calculation shows that the following orthogonality relations are satisfied:

$$
\int_{\mathbb{R}^{3}} \mathbf{r}_{i j} \cdot \mathbf{h}_{k l} \mathrm{~d} \xi=\delta_{i k} \delta_{j l}, \quad \int_{\mathbb{R}^{3}} \mathbf{r}_{i j} \cdot \mathbf{g}_{k} \mathrm{~d} \xi=\int_{\mathbb{R}^{3}} \mathbf{q}_{k} \cdot \mathbf{h}_{i j} \mathrm{~d} \xi=0 .
$$

Using these notations, any solution $\mathbf{w}$ of $(20)$ in $\mathrm{L}^{2}(m)$ can be decomposed as

$$
\mathbf{w}(\xi, \tau)=\sum_{i=1}^{3} \beta_{i}(\tau) \mathbf{f}_{i}(\xi)+\sum_{i=1}^{3} \gamma_{i}(\tau) \mathbf{g}_{i}(\xi)+\sum_{(i j) \in S} \zeta_{i j}(\tau) \mathbf{h}_{i j}(\xi)+\mathbf{R}(\xi, \tau)
$$


where $\beta_{i}(\tau)$ is given by $(38)$ and

$$
\gamma_{i}(\tau)=\int_{\mathbb{R}^{3}} \mathbf{q}_{i}(\xi) \cdot \mathbf{w}(\xi, \tau) \mathrm{d} \xi, \quad \zeta_{i j}(\tau)=\int_{\mathbb{R}^{3}} \mathbf{r}_{i j}(\xi) \cdot \mathbf{w}(\xi, \tau) \mathrm{d} \xi .
$$

Then $\mathbf{R}(\cdot, \tau)$ belongs to the subspace $\mathcal{W}_{2}$ of $\mathrm{L}^{2}(m)$ defined in (61), which coincides with the spectral subspace associated with the continuous spectrum $\left\{\lambda \in \mathbb{C} \mid \Re(\lambda) \leq \frac{1}{4}-\frac{m}{2}\right\}$ of the operator $\Lambda$. The coefficients $\gamma_{i}$ and $\zeta_{i j}$ satisfy the following evolution equations:

Lemma 4.1 Assume that $m>\frac{7}{2}$, and let $\mathbf{w} \in C^{0}\left([0, T], \mathrm{L}^{2}(m)\right)$ be a solution of (20). Then the coefficients $\gamma_{i}, \zeta_{i j}$ defined by (49) satisfy, for all $\tau \in[0, T]$,

$$
\begin{aligned}
\dot{\gamma}_{i}(\tau) & =-\frac{3}{2} \gamma_{i}(\tau), \quad i=1,2,3, \\
\dot{\zeta}_{i i}(\tau) & =-\frac{3}{2} \zeta_{i i}(\tau)+\frac{1}{2} \int_{\mathbb{R}^{3}}\left(v_{3}(\xi, \tau)^{2}-v_{i}(\xi, \tau)^{2}\right) \mathrm{d} \xi, \quad i=1,2, \\
\dot{\zeta}_{i j}(\tau) & =-\frac{3}{2} \zeta_{i j}(\tau)-\int_{\mathbb{R}^{3}} v_{i}(\xi, \tau) v_{j}(\xi, \tau) \mathrm{d} \xi, \quad 1 \leq i<j \leq 3,
\end{aligned}
$$

where $\mathbf{v}=\left(v_{1}, v_{2}, v_{3}\right)$ is the velocity field obtained from $\mathbf{w}$ via the Biot-Savart law (29).

Proof: We proceed as in the proof of Lemma 3.1. Differentiating (49) and integrating by parts, we find

$$
\begin{aligned}
\dot{\gamma}_{i} & =\int_{\mathbb{R}^{3}} \mathbf{q}_{i} \cdot(\Lambda \mathbf{w}+\operatorname{rot}(\mathbf{v} \wedge \mathbf{w})) \mathrm{d} \xi=-\frac{3}{2} \gamma_{i}+\int_{\mathbb{R}^{3}} \mathbf{p}_{i} \cdot\left(\frac{1}{2} \nabla|\mathbf{v}|^{2}-(\mathbf{v} \cdot \nabla) \mathbf{v}\right) \mathrm{d} \xi \\
& =-\frac{3}{2} \gamma_{i}+\int_{\mathbb{R}^{3}} \mathbf{v} \cdot\left((\mathbf{v} \cdot \nabla) \mathbf{p}_{i}\right) \mathrm{d} \xi=-\frac{3}{2} \gamma_{i},
\end{aligned}
$$

because $(\mathbf{v} \cdot \nabla) \mathbf{p}_{i}=\frac{1}{2} \mathbf{e}_{i} \wedge \mathbf{v} \perp \mathbf{v}$. Similarly, for all $(i j) \in S$, we find

$$
\dot{\zeta}_{i j}=-\frac{3}{2} \zeta_{i j}+\int_{\mathbb{R}^{3}} \mathbf{v} \cdot\left((\mathbf{v} \cdot \nabla) \operatorname{rot} \mathbf{r}_{i j}\right) \mathrm{d} \xi
$$

But $\operatorname{rot} \mathbf{r}_{i i}=\frac{1}{2}\left(\xi_{3} \mathbf{e}_{3}-\xi_{i} \mathbf{e}_{i}\right)$ and $\operatorname{rot} \mathbf{r}_{i j}=-\frac{1}{2}\left(\xi_{i} \mathbf{e}_{j}+\xi_{j} \mathbf{e}_{i}\right)$ for $i \neq j$, hence

$$
\mathbf{v} \cdot\left((\mathbf{v} \cdot \nabla) \operatorname{rot} \mathbf{r}_{i i}\right)=\frac{1}{2}\left(v_{3}^{2}-v_{i}^{2}\right), \quad \mathbf{v} \cdot\left((\mathbf{v} \cdot \nabla) \operatorname{rot} \mathbf{r}_{i j}\right)=-v_{i} v_{j} \quad(i \neq j) .
$$

This concludes the proof.

QED

The remainder $\mathbf{R}$ in (48) satisfies the equation

$$
\partial_{\tau} \mathbf{R}=\Lambda \mathbf{R}+Q_{2}((\mathbf{w} \cdot \nabla) \mathbf{v}-(\mathbf{v} \cdot \nabla) \mathbf{w}), \quad \operatorname{div} \mathbf{R}=0,
$$

where $Q_{2}: \mathrm{L}^{2}(m) \rightarrow \mathcal{W}_{2}$ is the spectral projection (for the operator $\Lambda$ ) onto the subspace $\mathcal{W}_{2}$, see Appendix A. Our next result describes the second order asymptotics of $\mathbf{w}(\tau)$ as $\tau \rightarrow \infty$. 
Theorem 4.2 Let $\frac{3}{2}<\nu<2, m>2 \nu+\frac{1}{2}$, and let $\mathbf{w} \in C^{0}\left([0, \infty), \mathrm{L}^{2}(m)\right)$ be the solution of (20) given by Theorem 2.3 with $\mu=1$. Then there exist constants $b_{i}, c_{i}, d_{i j}$, and $K_{5}$ such that $\left\|\mathbf{w}(\tau)-\mathbf{w}_{\text {app }}(\tau)\right\|_{m} \leq K_{5} \mathrm{e}^{-\nu \tau}\left\|\mathbf{w}_{0}\right\|_{m}$ for all $\tau \geq 0$, where

$$
\mathbf{w}_{a p p}(\xi, \tau)=\sum_{i=1}^{3} b_{i} \mathrm{e}^{-\tau} \mathbf{f}_{i}(\xi)+\sum_{i=1}^{3} c_{i} \mathrm{e}^{-\frac{3}{2} \tau} \mathbf{g}_{i}(\xi)+\sum_{(i j) \in S} d_{i j} \mathrm{e}^{-\frac{3}{2} \tau} \mathbf{h}_{i j}(\xi)
$$

Proof: If $\mathbf{w} \in C^{0}\left([0, \infty), \mathrm{L}^{2}(m)\right)$ is the solution of (20) given by Theorem 2.3 and $\mathbf{v}$ is the corresponding velocity field, we define $\beta_{i}, \gamma_{i}, \zeta_{i j}$, and $\mathbf{R}$ by (38), (48), (49). It is clear that $\beta_{i}(\tau)=b_{i} \mathrm{e}^{-\tau}$ and $\gamma_{i}(\tau)=c_{i} \mathrm{e}^{-\frac{3}{2} \tau}$, where

$$
b_{i}=\int_{\mathbb{R}^{3}} \mathbf{p}_{i}(\xi) \cdot \mathbf{w}_{0}(\xi) \mathrm{d} \xi, \quad c_{i}=\int_{\mathbb{R}^{3}} \mathbf{q}_{i}(\xi) \cdot \mathbf{w}_{0}(\xi) \mathrm{d} \xi, \quad i=1,2,3 .
$$

By Corollary 2.5, there exists $C_{1}>0$ such that $|\mathbf{v}(\tau)|_{2} \leq C_{1} \mathrm{e}^{-\tau}\left\|\mathbf{w}_{0}\right\|_{m}$ for all $\tau \geq 0$. Thus, it follows easily from Lemma 4.1 that $\left|\zeta_{i j}(\tau)-d_{i j} \mathrm{e}^{-\frac{3}{2} \tau}\right| \leq C_{2} \mathrm{e}^{-2 \tau}\left\|\mathbf{w}_{0}\right\|_{m}^{2}$ for all $\tau \geq 0$, where

$$
\begin{aligned}
& d_{i i}=\zeta_{i i}(0)+\frac{1}{2} \int_{0}^{\infty} \mathrm{e}^{\frac{3}{2} \tau} \int_{\mathbb{R}^{3}}\left(v_{3}(\xi, \tau)^{2}-v_{i}(\xi, \tau)^{2}\right) \mathrm{d} \xi \mathrm{d} \tau, \quad i=1,2, \\
& d_{i j}=\zeta_{i j}(0)-\int_{0}^{\infty} \mathrm{e}^{\frac{3}{2} \tau} \int_{\mathbb{R}^{3}} v_{i}(\xi, \tau) v_{j}(\xi, \tau) \mathrm{d} \xi \mathrm{d} \tau, \quad 1 \leq i<j \leq 3
\end{aligned}
$$

To bound the remainder $\mathbf{R}$, we proceed exactly as in the proof of Theorem 3.2. By Proposition A.3, there exists $C_{3}>0$ such that $\left\|\mathrm{e}^{\tau \Lambda} \mathbf{R}_{0}\right\|_{m} \leq C_{3} \mathrm{e}^{-\nu \tau}\left\|\mathbf{w}_{0}\right\|_{m}$ for all $\tau \geq 0$. Using the integral equation

$$
\mathbf{R}(\tau)=\mathrm{e}^{\tau \Lambda} \mathbf{R}_{0}+\int_{0}^{\tau} Q_{2} \boldsymbol{\Phi}(\tau-s, \mathbf{w}(s), \mathbf{v}(s)) \mathrm{d} s,
$$

together with the bound $\|\mathbf{w}(\tau)\|_{m} \leq K_{0} \mathrm{e}^{-\tau}\left\|\mathbf{w}_{0}\right\|_{m}$ given by Theorem 2.3, it is easy to show that $\|\mathbf{R}(\tau)\|_{m} \leq C_{4} \mathrm{e}^{-\nu \tau}\left\|\mathbf{w}_{0}\right\|_{m}$ for all $\tau \geq 0$.

QED

Corollary 4.3 Let $\frac{3}{2}<\nu<2$ and $m>2 \nu+\frac{1}{2}$. Let $\mathbf{w} \in C^{0}\left([0, \infty), \mathrm{L}^{2}(m)\right)$ be the solution of (20) given by Theorem 2.3 with $\mu=1$, and let $\mathbf{v}$ be the corresponding velocity field. There exists $K_{6}>0$ such that, for all $\tau \geq 1$,

$$
\begin{aligned}
& \left|\mathbf{w}(\tau)-\mathbf{w}_{a p p}(\tau)\right|_{p} \leq K_{6} \mathrm{e}^{-\nu \tau}\left\|\mathbf{w}_{0}\right\|_{m}, \quad 1 \leq p \leq \infty \\
& \left|\mathbf{v}(\tau)-\mathbf{v}_{a p p}(\tau)\right|_{q} \leq K_{6} \mathrm{e}^{-\nu \tau}\left\|\mathbf{w}_{0}\right\|_{m}, \quad 1 \leq q \leq \infty
\end{aligned}
$$

where

$$
\mathbf{v}_{\text {app }}(\xi, \tau)=\sum_{i=1}^{3} b_{i} \mathrm{e}^{-\tau} \mathbf{v}^{\mathbf{f}_{i}}(\xi)+\sum_{i=1}^{3} c_{i} \mathrm{e}^{-\frac{3}{2} \tau} \mathbf{v}^{\mathbf{g}_{i}}(\xi)+\sum_{(i j) \in S} d_{i j} \mathrm{e}^{-\frac{3}{2} \tau} \mathbf{v}^{\mathbf{h}_{i j}}(\xi)
$$

Moreover, the bounds (53), (54) hold for all $\tau \geq 0$ if $p \leq 2, q \leq 6$. 
In terms of the original variables, Corollary 4.3 shows that, for all $t \geq 1$,

$$
\begin{gathered}
\left|\boldsymbol{\omega}(t)-\boldsymbol{\omega}_{a p p}(t)\right|_{p} \leq C t^{-1-\nu+\frac{3}{2 p}}\left\|\boldsymbol{\omega}_{0}\right\|_{m}, \quad 1 \leq p \leq \infty \\
\left|\mathbf{u}(t)-\mathbf{u}_{a p p}(t)\right|_{q} \leq C t^{-\frac{1}{2}-\nu+\frac{3}{2 q}}\left\|\boldsymbol{\omega}_{0}\right\|_{m}, \quad 1 \leq q \leq \infty
\end{gathered}
$$

where $\boldsymbol{\omega}_{a p p}(x, t), \mathbf{u}_{a p p}(x, t)$ are the self-similar vector fields given by

$$
\begin{aligned}
\boldsymbol{\omega}_{a p p}(x, t) & =\frac{1}{1+t} \mathbf{w}_{a p p}\left(\frac{x}{\sqrt{1+t}}, \log (1+t)\right) \\
\mathbf{u}_{a p p}(x, t) & =\frac{1}{\sqrt{1+t}} \mathbf{v}_{a p p}\left(\frac{x}{\sqrt{1+t}}, \log (1+t)\right) .
\end{aligned}
$$

Remark 4.4 In contrast with the two-dimensional case, the second order asymptotic expansions of $\boldsymbol{\omega}(x, t)$ and $\mathbf{u}(x, t)$ contain only integer powers of $(1+t)^{-1 / 2}$, and not resonant terms of the form $(1+t)^{-\alpha} \log (1+t)$. However, following [13], one can show that such logarithmic terms do appear in the third order asymptotics. This is the reason why the case $\nu=2$ is excluded in Theorem 4.9. In fact, if $m>9 / 2$, the proof of Theorem 4.9 yields the estimate

$$
\left\|\mathbf{w}(\tau)-\mathbf{w}_{a p p}(\tau)\right\|_{m} \leq K_{5}(1+\tau) \mathrm{e}^{-2 \tau}\left\|\mathbf{w}_{0}\right\|_{m}, \quad \tau \geq 0
$$

which appears to be optimal.

Two prior papers which discuss the second order asymptotics of solutions of the NavierStokes equations are those of Carpio, [5], and Fujigaki and Miyakawa, [9]. As we demonstrate below, the results of Corollary 4.3 extend the results of these two references. The extension results from the fact that by imposing decay conditions on the initial velocity field (see the hypotheses of Theorem 0.6 in [5] and equation (1.2) of [9]) certain terms in the approximating velocity field $\mathbf{u}_{a p p}$ are forced to be zero. Thus, certain solutions of (2) of finite energy (i.e. of finite $L^{2}$ norm) whose asymptotics Corollary 4.3 allows us to compute are excluded from consideration by the decay conditions of [5] and [9]. This is a further reason that we feel it is more natural to impose decay conditions on the vorticity rather than the velocity. Note that in deriving the higher-order asymptotics in [9] (Theorem 2.2 (ii)) increasingly stringent decay conditions are imposed on the velocity which results in more and more terms in the asymptotics being zero. To compare the results of Corollary 4.3 with those of the previous references, first note that the requirement that $(1+|x|) \mathbf{v}_{0} \in L^{1}\left(\mathbb{R}^{3}\right)^{3}$ implies that that $b_{i}=0$ for $i=1,2,3$ by Corollary B.4. Furthermore, by Corollary B.5, the remaining coefficients in $\mathbf{v}_{a p p}$ satisfy

$$
c_{1}=b_{23}, c_{2}=b_{31}, c_{3}=b_{12}
$$

and

$$
d_{11}=\frac{1}{2}\left(c_{33}-c_{11}\right), d_{22}=\frac{1}{2}\left(c_{33}-c_{22}\right), d_{12}=-c_{12}, d_{23}=-c_{23}, d_{13}=-c_{13},
$$

where $b_{k \ell}$ and $c_{k \ell}$ are defined in (6). The expressions above for $d_{i j}$ follow easily from (19), (52) if one remembers that $\zeta_{i j}(0)=0$ by Corollary B.5. 
Now consider the term $\mathbf{A}(\xi)=\sum_{j=1}^{3} c_{j} \mathbf{v}^{\mathbf{g}_{j}}(\xi)$, in $\mathbf{v}_{\text {app }}$. Since $\mathbf{v}^{\mathbf{g}_{j}}=\mathbf{f}_{j}=\frac{1}{2}\left(\mathbf{e}_{j} \wedge \xi\right) G$, where $G(\xi)=(4 \pi)^{-3 / 2} \exp \left(-|\xi|^{2} / 4\right)$, this sum can be rewritten as

$$
\mathbf{A}(\xi)=\frac{1}{2} \sum_{j=1}^{3} c_{j}\left(\mathbf{e}_{j} \wedge \xi\right) G(\xi)=\frac{1}{2} G(\xi)(\mathbf{c} \wedge \xi)
$$

where $\mathbf{c}=\left(c_{1}, c_{2}, c_{3}\right)$. Examining this expression component-by-component, we see that

$$
A_{1}(\xi)=\frac{1}{2} G(\xi)\left(c_{2} \xi_{3}-c_{3} \xi_{2}\right)=\frac{1}{2} G(\xi)\left(b_{31} \xi_{3}-b_{12} \xi_{2}\right)=-\left(b_{31} \partial_{3} G+b_{21} \partial_{2} G\right)
$$

using the anti-symmetry of the $b_{j k}$. The other components are treated in like fashion and we find

$$
A_{j}(\xi)=-\sum_{k=1}^{3} b_{k j} \partial_{k} G(\xi), \quad j=1,2,3 .
$$

To treat the term $\mathbf{B}(\xi)=\sum_{(i j) \in S} d_{i j} \mathbf{v}^{\mathbf{h}_{i j}}(\xi)$, we note that using the expressions for $\mathbf{v}^{\mathbf{h}_{i j}}$ in Appendix B, one has

$$
\mathbf{v}^{\mathbf{h}_{i j}}=2 \partial_{i} \partial_{j}(\nabla \Phi)+\left(\partial_{i} G\right) \mathbf{e}_{j}+\left(\partial_{j} G\right) \mathbf{e}_{i},
$$

where $-\Delta \Phi=G$. Then using the expressions for $d_{i j}$ from above a straightforward computation shows that

$$
\mathbf{B}(\xi)=-\sum_{i, j=1}^{3} c_{i j}\left(\partial_{i} \partial_{j}(\nabla \Phi)+\left(\partial_{i} G\right) \mathbf{e}_{j}\right)
$$

In [9], the following notation is introduced:

$$
E_{t}(x)=\frac{1}{t^{3 / 2}} G\left(\frac{x}{\sqrt{t}}\right), \quad F_{\ell, j k}(x, t)=\frac{1}{t^{2}} F_{\ell, j k}\left(\frac{x}{\sqrt{t}}, 1\right), \quad x \in \mathbb{R}^{3}, \quad t>0,
$$

where $F_{\ell, j k}(\cdot, 1)=\partial_{k} \partial_{\ell}\left(\partial_{j} \Phi\right)+\left(\partial_{\ell} G\right) \delta_{j k}$. Using (56) and the expressions above for $\mathbf{A}(\xi)$ and $\mathbf{B}(\xi)$, it is easy to verify that the velocity field $\mathbf{u}_{a p p}$ in (55) satisfies

$$
\mathbf{e}_{j} \cdot \mathbf{u}_{a p p}(x, t-1)=-\sum_{k=1}^{3} b_{k j} \partial_{k} E_{t}(x)-\sum_{k, \ell=1}^{3} c_{k \ell} F_{\ell, j k}(x, t), \quad j=1,2,3 .
$$

Thus, written in terms of this notation, Corollary 4.3 implies:

Corollary 4.5 If in addition to the hypotheses of Corollary 4.3 , one assumes that the initial condition for the velocity field satisfies $\rho \mathbf{u}_{0} \in L^{1}\left(\mathbb{R}^{3}\right)^{3}$, then for $j=1,2,3$ one has the estimate

$$
\left|u_{j}(\cdot, t)+\sum_{k=1}^{3} b_{k j} \partial_{k} E_{t}(\cdot)+\sum_{k, \ell=1}^{3} c_{k \ell} F_{\ell, j k}(\cdot, t)\right|_{q} \leq C t^{-\frac{1}{2}-\nu+\frac{3}{2 q}}\left\|\boldsymbol{\omega}_{0}\right\|_{m}, \quad t \geq 1 .
$$

Comparing with equation (2.4) of [9] we see that this is compatible with the results of Fujigaki and Miyakawa. Rewriting the asymptotics in a slightly different way one finds that they also agree with Theorem 0.6 of [5]. 


\section{The strong-stable manifold of the origin}

In this section, we assume that $m>7 / 2$ and we consider in more detail the dynamics of (20) in the invariant subspace $\mathcal{W}_{1}$ of $\mathrm{L}^{2}(m)$ defined by (61). If $\mathbf{w}_{0} \in \mathcal{W}_{1}$ satisfies $\left\|\mathbf{w}_{0}\right\|_{m} \leq r_{0}$, where $r_{0}>0$ is as in Theorem 2.3, the solution $\mathbf{w}(\cdot, \tau)$ of (20) with initial data $\mathbf{w}_{0}$ can be decomposed as

$$
\mathbf{w}(\xi, \tau)=\sum_{i=1}^{3} \gamma_{i}(\tau) \mathbf{g}_{i}(\xi)+\sum_{(i j) \in S} \zeta_{i j}(\tau) \mathbf{h}_{i j}(\xi)+\mathbf{R}(\xi, \tau),
$$

where $\gamma_{i}, \zeta_{i j}$ are defined in (49) and $\mathbf{R}(\cdot, \tau)$ belongs to the subspace $\mathcal{W}_{2}$ of $\mathrm{L}^{2}(m)$. As for the velocity field, we have

$$
\mathbf{v}(\xi, \tau)=\sum_{i=1}^{3} \gamma_{i}(\tau) \mathbf{v}^{\mathbf{g}_{i}}(\xi)+\sum_{(i j) \in S} \zeta_{i j}(\tau) \mathbf{v}^{\mathbf{h}_{i j}}(\xi)+\mathbf{v}^{\mathbf{R}}(\xi, \tau) .
$$

Setting $b_{i}=0$ in Theorem 4.2, we see that $\|\mathbf{w}(\cdot, \tau)\|_{m}=\mathcal{O}\left(\mathrm{e}^{-\frac{3}{2} \tau}\right)$ as $\tau \rightarrow+\infty$. We now define the local strong-stable manifold of the origin by

$$
W_{s}^{l o c}=\left\{\mathbf{w}_{0} \in \mathrm{L}^{2}(m) \mid\left\|\mathbf{w}_{0}\right\|_{m} \leq r_{0}, \lim _{\tau \rightarrow \infty} \mathrm{e}^{\frac{3}{2} \tau}\left\|\Phi_{\tau} \mathbf{w}_{0}\right\|_{m}=0\right\},
$$

where $\Phi_{\tau} \mathbf{w}_{0}=\mathbf{w}(\tau)$ is the solution of $(20)$ in $L^{2}(m)$ with initial data $\mathbf{w}_{0}$. It is clear from Theorem 3.2 that $W_{s}^{\text {loc }} \subset \mathcal{W}_{1}$. On the other hand, it follows from Lemma 4.1 that $W_{s}^{\text {loc }} \not \subset \mathcal{W}_{2}$. Indeed, the inclusion $W_{s}^{\text {loc }} \subset \mathcal{W}_{2}$ would mean that the integrals in the righthand side of (50) vanish identically for vorticities $\mathbf{w} \in \mathcal{W}_{2}$, but it is easy to verify that this is not the case.

By construction, $\mathcal{W}_{1}=\mathcal{W}_{2} \oplus \mathcal{V}$, where $\mathcal{V}$ is the eight-dimensional space spanned by the vector fields $\mathbf{g}_{i}$ for $i=1,2,3$ and $\mathbf{h}_{i j}$ for $(i j) \in S$. Using invariant manifold theory as in [13], it is rather straightforward to show that $W_{s}^{\text {loc }}$ is a smooth submanifold of $\mathcal{W}_{1}$ which is tangent at the origin to the subspace $\mathcal{W}_{2}$. In other words, there exists a smooth function $f: \mathcal{W}_{2} \rightarrow \mathcal{V}$ satisfying $f(0)=0, f^{\prime}(0)=0$, and such that $W_{s}^{\text {loc }}=G(f) \cap B\left(r_{0}\right)$, where

$$
G(f)=\left\{\mathbf{w}+f(\mathbf{w}) \mid \mathbf{w} \in \mathcal{W}_{2}\right\} \subset \mathcal{W}_{1}, \quad B\left(r_{0}\right)=\left\{\mathbf{w} \in \mathcal{W}_{1} \mid\|\mathbf{w}\|_{m} \leq r_{0}\right\} .
$$

In particular, the manifold $W_{s}^{\text {loc }}$ is of codimension 8 in $\mathcal{W}_{1}$, hence of codimension 11 in $\mathrm{L}^{2}(m)$. By construction, $W_{s}^{l o c}$ is locally positively invariant in the following sense: if $\mathbf{w}_{0} \in W_{s}^{\text {loc }}$, then $\mathbf{w}(\tau)=\Phi_{\tau} \mathbf{w}_{0} \in W_{s}^{\text {loc }}$ as long as $\mathbf{w}(\tau) \in B\left(r_{0}\right)$. If in addition $\left\|\mathbf{w}_{0}\right\|_{m} \leq r_{0} / K_{0}$, where $K_{0}$ is as in Theorem 2.3, then $\mathbf{w}(\tau) \in W_{s}^{\text {loc }}$ for all $\tau \geq 0$ and $\mathrm{e}^{\frac{3}{2} \tau}\|\mathbf{w}(\tau)\|_{m} \rightarrow 0$ as $\tau \rightarrow+\infty$.

The following result is characterization of the local strong-stable manifold.

Proposition 5.1 Fix $m>7 / 2$, and assume that $\mathbf{w}_{0} \in \mathcal{W}_{1} \subset \mathrm{L}^{2}(m)$ satisfies $\left\|\mathbf{w}_{0}\right\|_{m} \leq r_{0}$, where $r_{0}>0$ is as in Theorem 2.3. Let $w(\xi, \tau)$ be the solution of (20) with initial data 


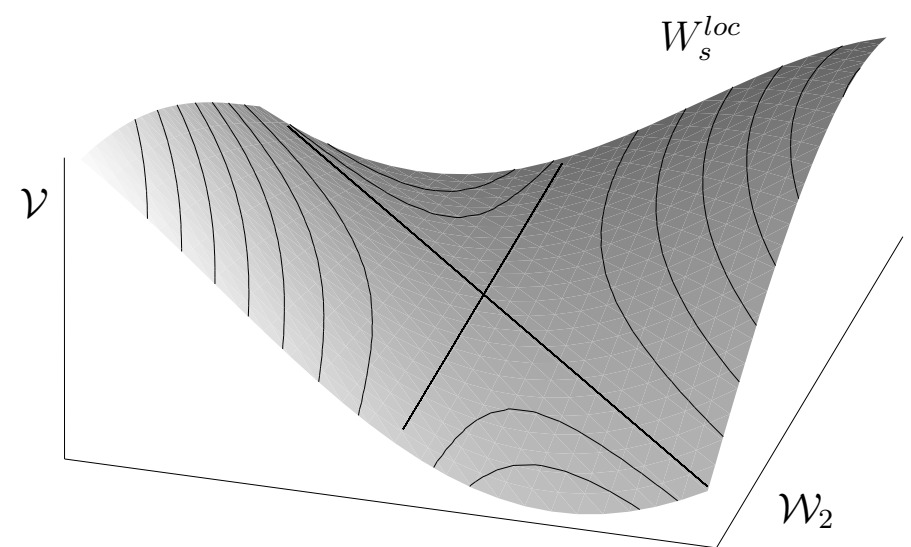

Fig. 2: A schematic picture of the local strong-stable manifold $W_{s}^{\text {loc }}$ (shaded surface). The horizontal plane is the infinite-dimensional subspace $\mathcal{W}_{2}$, and the vertical axis the eight-dimensional space $\mathcal{V}$. The intersection $W_{s}^{\text {loc }} \cap \mathcal{W}_{2}$, which is also infinite-dimensional, is represented by two line segments.

$\mathbf{w}_{0}$, and let $\mathbf{v}(\xi, \tau)$ be the corresponding velocity field. Define the functions $\gamma_{i}(\tau), \zeta_{i j}(\tau)$ by (49) and the coefficients $c_{k \ell}$ by (6), with

$$
\mathbf{u}(x, t)=\frac{1}{\sqrt{1+t}} \mathbf{v}\left(\frac{x}{\sqrt{1+t}}, \log (1+t)\right) .
$$

Then the following three statements are equivalent:

1) $\lim _{\tau \rightarrow \infty} \mathrm{e}^{\frac{3}{2} \tau}\|\mathbf{w}(\cdot, \tau)\|_{m}=0$, namely $\mathbf{w}_{0} \in W_{s}^{\text {loc }}$.

2) $\lim _{t \rightarrow \infty} t^{5 / 4}|\mathbf{u}(\cdot, t)|_{2}=0$.

3) $\gamma_{1}(0)=\gamma_{2}(0)=\gamma_{3}(0)=0$,

$\zeta_{11}(0)=\frac{1}{2}\left(c_{11}-c_{33}\right), \zeta_{22}(0)=\frac{1}{2}\left(c_{22}-c_{33}\right), \zeta_{12}(0)=c_{12}, \zeta_{13}(0)=c_{13}, \zeta_{23}(0)=c_{23}$.

Proof: We apply Theorem 4.2 with $\frac{3}{2}<\nu<\frac{m}{2}-\frac{1}{4}$. Since $\mathbf{w}_{0} \in \mathcal{W}_{1}$, we have $b_{i}=0$ for $i=1,2,3$, so that $\|\mathbf{w}(\tau)\|_{m}=\mathcal{O}\left(\mathrm{e}^{-\frac{3}{2} \tau}\right)$ as $\tau \rightarrow+\infty$. We shall show that statements 1$)$, $2), 3)$ in Proposition 5.1 are all equivalent to

4) $c_{i}=0$ for $i=1,2,3$ and $d_{i j}=0$ for all $(i j) \in S$.

Indeed, it is clear from (51) that $\left\|\mathbf{w}_{\text {app }}(\tau)\right\|_{m}=K \mathrm{e}^{-\frac{3}{2} \tau}$, where $K=0$ if and only if 4) holds. Thus 1) $\Leftrightarrow 4$ ) by Theorem 4.2. Similarly, it follows from (56) that $\left|\mathbf{u}_{a p p}(t)\right|_{2}=$ $K^{\prime}(1+t)^{-5 / 4}$, where $K^{\prime}=0$ if and only if 4$)$ holds. Since $\left|\mathbf{u}(t)-\mathbf{u}_{a p p}(t)\right|_{2} \leq C(1+t)^{\frac{1}{4}-\nu}$ by (55), we conclude that 2) $\Leftrightarrow 4$ ). Finally, using (6), (52) and the change of variables (59), we obtain the relations

$$
\begin{aligned}
d_{i i} & =\zeta_{i i}(0)+\frac{1}{2}\left(c_{33}-c_{i i}\right), \quad i=1,2, \\
d_{i j} & =\zeta_{i j}(0)-c_{i j}, \quad 1 \leq i<j \leq 3 .
\end{aligned}
$$

We also know that $c_{i}=\gamma_{i}(0)$ for $i=1,2,3$. Therefore, 3$\left.) \Leftrightarrow 4\right)$.

Using Proposition 5.1, it is easy to prove Theorem 1.1] in the case where $\mathbf{w}_{0}=\operatorname{rot}\left(\mathbf{u}_{0}\right)$ satisfies $\left\|\mathbf{w}_{0}\right\|_{m} \leq r_{0}$ for some $m>7 / 2$. Indeed, let $\mathbf{w}(\xi, \tau)$ be the solution of (20) 
with initial data $\mathbf{w}_{0}$, and define $\gamma_{i}(\tau), \zeta_{i j}(\tau)$ by (49). By Corollary B.5, the assumption $\rho \mathbf{u}_{0} \in L^{1}\left(\mathbb{R}^{3}\right)^{3}$ implies that $\mathbf{w}_{0} \in \mathcal{W}_{1}$ (so that $\mathbf{w}(\tau) \in \mathcal{W}_{1}$ for all $\tau \geq 0$ ) and that $\zeta_{i j}(0)=0$ for all $(i j) \in S$.

a) Assume first that (7) holds, namely $\mathbf{w}_{0} \in W_{s}^{\text {loc }}$. Then point 3) in Proposition 5.1 shows that $c_{11}=c_{22}=c_{33}$ and $c_{i j}=0$ if $i \neq j$, hence the matrix $\left(c_{k \ell}\right)$ is scalar. In addition, since $\gamma_{i}(0)=0$, we have $b_{k \ell}=0$ by Corollary B.5. This proves (8).

b) Conversely, assume that (8) holds. Then $\gamma_{i}(0)=0$ by Corollary B.5, and since $\zeta_{i i}(0)=$ $0=\frac{1}{2}\left(c_{i i}-c_{33}\right)$ for $i=1,2, \zeta_{i j}(0)=0=c_{i j}$ for $i \neq j$, it follows from Proposition 5.1 that $\mathbf{w}_{0} \in W_{s}^{\text {loc }}$. This concludes the argument.

QED

As is clear from this proof, if $\mathbf{w}_{0}$ lies in $W_{s}^{l o c}$ and if the corresponding velocity field $\mathbf{v}_{0}$ satisfies $\rho \mathbf{v}_{0} \in L^{1}\left(\mathbb{R}^{3}\right)^{3}$, then necessarily $\mathbf{w}_{0} \in \mathcal{W}_{2}$. Thus, from our point of view, Theorem 1.1 is a characterization of the noninvariant set $W_{s}^{\text {loc }} \cap \mathcal{W}_{2}$, and not of $W_{s}^{\text {loc }}$ itself. As was already observed, $W_{s}^{\text {loc }}$ is not contained in $\mathcal{W}_{2}$, and it is not a priori obvious that $W_{s}^{\text {loc }} \cap \mathcal{W}_{2} \neq\{0\}$ ! In fact, using a nice argument due to L. Brandolese, it turns out that $W_{s}^{\text {loc }} \cap \mathcal{W}_{2}$ is infinite-dimensional. Following $[1]$, we say that a vector field $\mathbf{u}: \mathbb{R}^{3} \rightarrow \mathbb{R}^{3}$ is symmetric if it satisfies the following two properties:

A) $u_{1}\left(x_{1}, x_{2}, x_{3}\right)=u_{2}\left(x_{3}, x_{1}, x_{2}\right)=u_{3}\left(x_{2}, x_{3}, x_{1}\right)$ for all $x=\left(x_{1}, x_{2}, x_{3}\right) \in \mathbb{R}^{3}$.

B) For all $i \in\{1,2,3\}, u_{i}\left(x_{1}, x_{2}, x_{3}\right)$ is an odd function of $x_{i}$ and an even function of $x_{j}$ for all $j \neq i$.

If $\mathbf{u}$ is symmetric, then $\Delta \mathbf{u}$ and $(\mathbf{u} \cdot \nabla) \mathbf{u}$ are also symmetric. This implies, roughly speaking, that the space of symmetric velocity fields is invariant under the Navier-Stokes evolution (whenever defined).

Assume now that $\mathbf{v}: \mathbb{R}^{3} \rightarrow \mathbb{R}^{3}$ is symmetric and that the vorticity $\mathbf{w}=\operatorname{rot} \mathbf{v}$ belongs to $\mathrm{L}^{2}(m)$ for some $m>7 / 2$. Then $\mathbf{w}$ satisfies:

$\left.\mathrm{A}^{\prime}\right) w_{1}\left(\xi_{1}, \xi_{2}, \xi_{3}\right)=w_{2}\left(\xi_{3}, \xi_{1}, \xi_{2}\right)=w_{3}\left(\xi_{2}, \xi_{3}, \xi_{1}\right)$ for all $\xi \in \mathbb{R}^{3}$.

B') For all $i \in\{1,2,3\}, w_{i}\left(\xi_{1}, \xi_{2}, \xi_{3}\right)$ is an even function of $\xi_{i}$ and an odd function of $\xi_{j}$ for all $j \neq i$.

Using these properties together with (38), (49), we discover that $\beta_{i}=\gamma_{i}=\zeta_{i j}=0$ for all $i, j$, hence $\mathbf{w} \in \mathcal{W}_{2}$. On the other hand, it is clear that the integrals in the right-hand side of (50) vanish identically if $\mathbf{v}$ is symmetric, so that $c_{k \ell}=0$ in (6). Thus, if $\|\mathbf{w}\|_{m} \leq r_{0}$, it follows from Proposition 5.1 that $\mathbf{w} \in W_{s}^{\text {loc }}$. Summarizing, we have shown:

$$
W_{s}^{\text {loc }} \cap \mathcal{W}_{2} \supset\left\{\mathbf{w} \in \mathrm{L}^{2}(m) \mid\|\mathbf{w}\|_{m} \leq r_{0}, \mathbf{w}=\operatorname{rot} \mathbf{v} \text { with } \mathbf{v} \text { symmetric }\right\} .
$$

We conclude this section with a somewhat surprising observation. Let $\Psi_{t}$ be the local semiflow defined by the vorticity equation $(9)$ in $\mathrm{L}^{2}(m)$ for $m>7 / 2$. If $\boldsymbol{\omega} \in \mathrm{L}^{2}(m)$, let $\mathbf{U}_{\omega}$ be the velocity field obtained from $\boldsymbol{\omega}$ via the Biot-Savart law (10). Then

$$
W_{s}^{l o c}=\left\{\left.\boldsymbol{\omega}_{0} \in \mathrm{L}^{2}(m)\left|\left\|\boldsymbol{\omega}_{0}\right\|_{m} \leq r_{0}, \lim _{t \rightarrow+\infty} t^{5 / 4}\right| \mathbf{U}_{\Psi_{t} \omega_{0}}\right|_{2}=0\right\}
$$

This characterization follows from the equivalence 1) $\Leftrightarrow 2$ ) in Proposition 5.1 and from the fact that the change of variables (18), (19) reduces to the identity when $t=0$. As a consequence, $W_{s}^{\text {loc }}$ is locally invariant under both semiflows $\Psi_{t}$ and $\Phi_{\tau}$, although the orbits of the same initial point under $\Psi_{t}$ and $\Phi_{\tau}$ are of course different! This curious property originates in the fact that in both the original and rescaled variables the strong-stable 
manifold can be characterized in terms of the decay rate (as time goes to infinity) of the solutions lying in it, see [13] for a more detailed discussion. In concrete terms, the observation above implies that the picture of $W_{s}^{l o c}$ in Fig. 2 is not affected at all when we return to the original variables using (18).

\section{A Spectrum of the operator $\Lambda$}

In Appendix A of [13], we study in detail the linear operator

$$
\mathcal{L}=\Delta_{\xi}+\frac{1}{2} \xi \cdot \nabla_{\xi}+\frac{N}{2}, \quad \xi \in \mathbb{R}^{N}, \quad N \geq 1
$$

acting on the function $L^{2}(m)=\left\{f \in L^{2}\left(\mathbb{R}^{N}\right) \mid\|f\|_{m}<\infty\right\}$, where

$$
\|f\|_{m}=\left(\int_{\mathbb{R}^{N}}(1+|\xi|)^{2 m}|f(\xi)|^{2} \mathrm{~d} \xi\right)^{1 / 2} .
$$

In particular, we determine exactly the spectrum of $\mathcal{L}$ :

Theorem A.1 [13] Fix $m \geq 0$, and let $\mathcal{L}$ be the linear operator (60) in $L^{2}(m)$, defined on its maximal domain. Then the spectrum of $\mathcal{L}$ is

$$
\sigma(\mathcal{L})=\left\{\lambda \in \mathbb{C} \mid \Re(\lambda) \leq \frac{N}{4}-\frac{m}{2}\right\} \cup\left\{-\frac{k}{2} \mid k \in \mathbb{N}\right\}
$$

Moreover, if $m>\frac{N}{2}$ and if $k \in \mathbb{N}$ satisfies $k+\frac{N}{2}<m$, then $\mu_{k}=-\frac{k}{2}$ is an isolated eigenvalue of $\mathcal{L}$, with multiplicity $\left(\begin{array}{c}N+k-1 \\ k\end{array}\right)$.

The eigenfunctions corresponding to the isolated eigenvalues $\mu_{k}=-\frac{k}{2}$ can be computed explicitly. Moreover, it is shown in [13] that $\mathcal{L}$ generates a $C_{0}$ semigroup $\mathrm{e}^{\tau \mathcal{L}}$ in $L^{2}(m)$, and sharp estimates are obtained for the norm of $\mathrm{e}^{\tau \mathcal{L}}$ in various spectral subspaces of $L^{2}(m)$.

In this section, we adapt the results in 13 to the particular case where $N=3$ and $\mathcal{L} \equiv \Lambda+\frac{1}{2}$ acts on the space of divergence free vector fields $\mathrm{L}^{2}(m)$ defined in (24). Remark that $\operatorname{div}(\Lambda \mathbf{f})=\mathcal{L} \operatorname{div}(\mathbf{f})$, so that $\Lambda$ preserves the divergence free condition. The analogue of Theorem A.1 is:

Theorem A.2 Fix $m \geq 0$, and let $\Lambda$ be the linear operator (21) in $\mathrm{L}^{2}(m)$, defined on its maximal domain. Then the spectrum of $\Lambda$ is

$$
\sigma(\Lambda)=\left\{\lambda \in \mathbb{C} \mid \Re(\lambda) \leq \frac{1}{4}-\frac{m}{2}\right\} \cup\left\{-\frac{k+1}{2} \mid k \in \mathbb{N}^{*}\right\}
$$

where $\mathbb{N}^{*}=\mathbb{N} \backslash\{0\}$. Moreover, if $m>\frac{5}{2}$ and if $k \in \mathbb{N}^{*}$ satisfies $k+\frac{3}{2}<m$, then $\lambda_{k}=-\frac{k+1}{2}$ is an isolated eigenvalue of $\mathcal{L}$, with multiplicity $k(k+2)$. 
Proof: We first discuss the discrete spectrum of $\Lambda$. Fix $k \in \mathbb{N}$, and take $\alpha=\left(\alpha_{1}, \alpha_{2}, \alpha_{3}\right) \in$ $\mathbb{N}^{3}$ such that $|\alpha|=\alpha_{1}+\alpha_{2}+\alpha_{3}=k$. Then the Hermite function $\phi_{\alpha}: \mathbb{R}^{3} \rightarrow \mathbb{R}$ defined by

$$
\phi_{\alpha}=\partial^{\alpha} G \equiv \partial_{1}^{\alpha_{1}} \partial_{2}^{\alpha_{2}} \partial_{3}^{\alpha_{3}} G, \quad \text { where } G(\xi)=\frac{1}{(4 \pi)^{3 / 2}} \mathrm{e}^{-|\xi|^{2} / 4}
$$

is an eigenfunction of $\mathcal{L}$ with eigenvalue $-\frac{k}{2}$. Let $E_{k}=\operatorname{span}\left\{\phi_{\alpha}\left|\alpha \in \mathbb{N}^{3},\right| \alpha \mid=k\right\}$ and

$$
\mathcal{E}_{k}=\left\{\mathbf{f}=\left(f_{1}, f_{2}, f_{3}\right) \mid \operatorname{div} \mathbf{f}=0, f_{i} \in E_{k} \text { for } i=1,2,3\right\} .
$$

By construction, $\mathcal{E}_{k} \subset \mathrm{L}^{2}(m)$ for all $m \geq 0$, and any $\mathbf{f} \in \mathcal{E}_{k}$ satisfies $\Lambda \mathbf{f}=-\frac{k+1}{2} \mathbf{f}$. Moreover, using the characterization of $\mathcal{E}_{k}$ in Fourier variables (see [13]), it is not difficult to show that $\operatorname{dim}\left(\mathcal{E}_{k}\right)=k(k+2)$. In particular, for any $k \in \mathbb{N}^{*}, \lambda_{k}=-\frac{k+1}{2}$ is an eigenvalue of $\Lambda$ with multiplicity (at least) $k(k+2)$.

Next, fix $\lambda \in \mathbb{C}$ such that $\Re(\lambda)<1 / 4$ and $-(\lambda+1) \notin \mathbb{N}$. Proceeding as in [13], it is easy to verify that the function $\psi_{\lambda}: \mathbb{R}^{3} \rightarrow \mathbb{R}$ defined in Fourier variables by

$$
\hat{\psi}_{\lambda}(p)=|p|^{-2(\lambda+1)} \mathrm{e}^{-|p|^{2}}\left(-\mathrm{i} p_{2}, \mathrm{i} p_{1}, 0\right)
$$

satisfies $\Lambda \psi_{\lambda}=\lambda \psi_{\lambda}$ and $\operatorname{div} \psi_{\lambda}=0$. Moreover, $\psi_{\lambda} \in \mathrm{L}^{2}(m)$ if and only if $\Re(\lambda)<\frac{1}{4}-\frac{m}{2}$. This shows that $\sigma(\Lambda) \supset\left\{\lambda \in \mathbb{C} \mid \Re(\lambda) \leq \frac{1}{4}-\frac{m}{2}\right\}$.

Now, fix $n \in \mathbb{Z}$ and assume that $m \geq 0, m>n+\frac{3}{2}$. Let

$$
W_{n}=\left\{f \in L^{2}(m) \mid \int_{\mathbb{R}^{3}} \xi^{\alpha} f(\xi) \mathrm{d} \xi=0 \text { for all } \alpha \in \mathbb{N}^{3} \text { with }|\alpha| \leq n\right\} .
$$

In particular, $W_{n}=L^{2}(m)$ if $n<0$. We define closed subspaces $\mathcal{V}_{n}, \mathcal{W}_{n}$ of $\mathrm{L}^{2}(m)$ by $\mathcal{V}_{n}=\oplus_{k=1}^{n} \mathcal{E}_{k}$ and

$$
\mathcal{W}_{n}=\left\{\mathbf{f} \in \mathrm{L}^{2}(m) \mid f_{i} \in W_{n} \text { for } i=1,2,3\right\} .
$$

By definition, $\mathcal{V}_{n}=\{0\}$ and $\mathcal{W}_{n}=\mathrm{L}^{2}(m)$ if $n \leq 0$. (We recall that any $\mathbf{f} \in \mathrm{L}^{2}(m)$ with $m>\frac{3}{2}$ satisfies $\int_{\mathbb{R}^{3}} \mathbf{f}(\xi) \mathrm{d} \xi=0$ as a consequence of the divergence free condition; hence $\mathcal{W}_{0}=\mathrm{L}^{2}(m)$.) Using again the characterization of $\mathrm{L}^{2}(m)$ in Fourier variables, it is easy to verify that $\mathrm{L}^{2}(m)=\mathcal{V}_{n} \oplus \mathcal{W}_{n}$. Let $P_{n}: \mathrm{L}^{2}(m) \rightarrow \mathrm{L}^{2}(m)$ be the (unique) continuous projection satisfying range $\left(P_{n}\right)=\mathcal{V}_{n}, \operatorname{ker}\left(P_{n}\right)=\mathcal{W}_{n}$, and let $Q_{n}=\mathbf{1}-P_{n}$. In particular, $P_{n}=0$ and $Q_{n}=\mathbf{1}$ for all $n \in \mathbb{Z}, n \leq 0$. The following estimates on the semigroup $\mathrm{e}^{\tau \Lambda}=\mathrm{e}^{-\tau / 2} \mathrm{e}^{\tau \mathcal{L}}$ are proved in [13] (Proposition A.2):

Proposition A.3 Let $a(\tau)=1-\mathrm{e}^{-\tau}, \tau \geq 0$.

(a) Fix $m \geq 0$, and take $n \in \mathbb{Z}$ such that $n+\frac{3}{2}<m \leq n+\frac{5}{2}$. For all $\alpha \in \mathbb{N}^{3}$ and all $\epsilon>0$, there exists $C>0$ such that

$$
\left\|\partial^{\alpha} \mathrm{e}^{\tau \Lambda} f\right\|_{m} \leq \frac{C}{a(\tau)^{|\alpha| / 2}} \mathrm{e}^{\frac{\tau}{2}\left(\frac{1}{2}-m+\epsilon\right)}\|f\|_{m}, \quad \tau>0,
$$

for all $f \in W_{n} \subset L^{2}(m)$.

(b) Fix $n \in \mathbb{N} \cup\{-1\}$, and take $m \in \mathbb{R}$ such that $m>n+\frac{5}{2}$. For all $\alpha \in \mathbb{N}^{3}$ and all $\epsilon>0$, there exists $C>0$ such that

$$
\left\|\partial^{\alpha} \mathrm{e}^{\tau \Lambda} f\right\|_{m} \leq \frac{C}{a(\tau)^{|\alpha| / 2}} \mathrm{e}^{-\frac{n+2}{2} \tau}\|f\|_{m}, \quad \tau>0
$$

for all $f \in W_{n} \subset L^{2}(m)$. 
If $m$ and $n$ are as in part (a) of Proposition A.3, it follows from (62) that

$$
\left\|\mathrm{e}^{\tau \Lambda} Q_{n} \mathbf{f}\right\|_{m} \leq C \mathrm{e}^{\frac{\tau}{2}\left(\frac{1}{2}-m+\epsilon\right)}\|\mathbf{f}\|_{m}, \quad \tau \geq 0
$$

for all $\mathbf{f} \in \mathrm{L}^{2}(m)$. By the Hille-Yosida theorem, this implies that $\sigma\left(\Lambda Q_{n}\right) \subset\{\lambda \in$ $\left.\mathbb{C} \mid \Re(\lambda) \leq \frac{1}{4}-\frac{m}{2}\right\}$. On the other hand, by construction, we have $\sigma\left(\Lambda P_{n}\right)=\emptyset$ if $n \leq 0$ and $\sigma\left(\Lambda P_{n}\right)=\left\{-1 ;-\frac{3}{2} ; \ldots ;-\frac{n+1}{2}\right\}$ if $n \in \mathbb{N}^{*}$. In particular, $\sigma\left(\Lambda P_{n}\right) \cap \sigma\left(\Lambda Q_{n}\right)=\emptyset$, hence the multiplicity of the eigenvalue $\lambda_{k}(k=1, \ldots, n)$ is exactly $k(k+2)$. Finally, since $\sigma(\Lambda) \subset \sigma\left(\Lambda P_{n}\right) \cup \sigma\left(\Lambda Q_{n}\right)$, we have

$$
\sigma(\Lambda) \subset\left\{\lambda \in \mathbb{C} \mid \Re(\lambda) \leq \frac{1}{4}-\frac{m}{2}\right\} \cup\left\{-\frac{k+1}{2} \mid k \in \mathbb{N}^{*}\right\}
$$

This concludes the proof of Theorem A.2.

QED

The estimates in Proposition A.3 can be generalized to weighted $L^{p}$ spaces with $p \neq 2$. For our purposes in this paper, the following result will be sufficient:

Proposition A.4 Let $1 \leq q \leq p \leq \infty, m \geq 0$ and $T>0$. For all $\alpha \in \mathbb{N}^{3}$, there exists $C>0$ such that, for all $f \in L^{q}(m)$,

$$
\left|\rho^{m} \partial^{\alpha} \mathrm{e}^{\tau \Lambda} f\right|_{p} \leq \frac{C}{a(\tau)^{\frac{3}{2}\left(\frac{1}{q}-\frac{1}{p}\right)+\frac{|\alpha|}{2}}}\left|\rho^{m} f\right|_{q}, \quad 0<\tau \leq T,
$$

where $\rho(\xi)=1+|\xi|$.

Proof: See [13], Proposition A.5.

\section{B Bounds on the velocity field}

We first list a few identities that are satisfied by the vorticity $\mathbf{w}$ as a consequence of the divergence free condition. If $\mathbf{w} \in \mathrm{L}^{1}\left(\mathbb{R}^{3}\right)^{3}$, then

$$
\int_{\mathbb{R}^{3}} w_{i}(\xi) \mathrm{d} \xi=0 \quad \text { for all } i \in\{1,2,3\}
$$

because $w_{i}=\operatorname{div}\left(\xi_{i} \mathbf{w}\right)$. This fact is not hard to prove in Fourier variables, but has been overlooked in some papers on the subject until recently. If in addition $\rho \mathbf{w} \in L^{1}\left(\mathbb{R}^{3}\right)^{3}$ (where $\rho(\xi)=1+|\xi|$ ), then

$$
\int_{\mathbb{R}^{3}}\left(\xi_{i} w_{j}(\xi)+\xi_{j} w_{i}(\xi)\right) \mathrm{d} \xi=0 \quad \text { for all } i, j \in\{1,2,3\}
$$

because $\xi_{i} w_{j}+\xi_{j} w_{i}=\operatorname{div}\left(\xi_{i} \xi_{j} \mathbf{w}\right)$. Thus only three first-order moments of $\mathbf{w}$ (out of nine) are possibly nonzero. Finally, if we assume that $\rho^{2} \mathbf{w} \in L^{1}\left(\mathbb{R}^{3}\right)^{3}$, then

$$
\int_{\mathbb{R}^{3}}\left(\xi_{i} \xi_{j} w_{k}(\xi)+\xi_{j} \xi_{k} w_{i}(\xi)+\xi_{k} \xi_{i} w_{j}(\xi)\right) \mathrm{d} \xi=0 \quad \text { for all } i, j, k \in\{1,2,3\}
$$

because $\xi_{i} \xi_{j} w_{k}+\xi_{j} \xi_{k} w_{i}+\xi_{k} \xi_{i} w_{j}=\operatorname{div}\left(\xi_{i} \xi_{j} \xi_{k} \mathbf{w}\right)$. This means that only eight second-order moments of $\mathbf{w}$ (out of eighteen) are possibly nonzero. 
Next, we give explicit formulas for the velocity fields corresponding, via the Biot-Savart law (10), to the first eigenfunctions of the linear operator $\Lambda$ acting on $\mathrm{L}^{2}(m)$.

1) If $m>5 / 2$, the first eigenvalue $\lambda_{2}=-1$ has multiplicity three. A basis of eigenfunctions is $\left\{\mathbf{f}_{1}, \mathbf{f}_{2}, \mathbf{f}_{3}\right\}$, where $\mathbf{f}_{i}=\mathbf{p}_{i} G$ and $\mathbf{p}_{1}, \mathbf{p}_{2}, \mathbf{p}_{3}$ are defined in (36). The corresponding velocity fields are

$$
\mathbf{v}^{\mathbf{f}_{i}}=\partial_{i}(\nabla \Phi)+G \mathbf{e}_{i}, \quad i=1,2,3
$$

where

$$
\Phi(\xi)=\frac{1}{(4 \pi)^{3 / 2}} \frac{2}{|\xi|} \int_{0}^{|\xi|} \mathrm{e}^{-z^{2} / 4} \mathrm{~d} z, \quad \xi \in \mathbb{R}^{3} \backslash\{0\} .
$$

Remark that $-\Delta \Phi=G$, so that $\operatorname{div} \mathbf{v}^{\mathbf{f}_{i}}=0$ and $\operatorname{rot} \mathbf{v}^{\mathbf{f}_{i}}=\operatorname{rot}\left(G \mathbf{e}_{i}\right)=\mathbf{f}_{i}$. A direct calculation shows that $\mathbf{v}^{\mathbf{f}_{i}} \sim|\xi|^{-3}$ as $|\xi| \rightarrow \infty$, hence $\mathbf{v}^{\mathbf{f}_{i}} \notin \mathrm{L}^{1}\left(\mathbb{R}^{3}\right)$.

2) If $m>7 / 2$, the second eigenvalue $\lambda_{3}=-3 / 2$ has multiplicity eight. A convenient basis of eigenfunctions is given by $\left\{\mathbf{g}_{i}\right\}_{i=1,2,3}$ and $\left\{\mathbf{h}_{i j}\right\}_{(i j) \in S}$, where $\mathbf{g}_{i}$ are defined in (44) and $\mathbf{h}_{i j}$ in (46). (We recall that $S=\{(11),(12),(13),(22),(23)\}$.) The corresponding velocity fields read

$$
\mathbf{v}^{\mathbf{g}_{i}}=\mathbf{f}_{i}, \quad \mathbf{v}^{\mathbf{h}_{i j}}=\partial_{i} \mathbf{v}^{\mathbf{f}_{j}}+\partial_{j} \mathbf{v}^{\mathbf{f}_{i}} .
$$

Clearly, $\mathbf{v}^{\mathbf{g}_{i}}(\xi)$ has a Gaussian decay as $|\xi| \rightarrow \infty$, whereas $\mathbf{v}^{\mathbf{h}_{i j}}(\xi) \sim|\xi|^{-4}$. In particular, $\mathbf{v}^{\mathbf{h}_{i j}} \in \mathrm{L}^{1}\left(\mathbb{R}^{3}\right)$, but $\rho \mathbf{v}^{\mathbf{h}_{i j}} \notin \mathrm{L}^{1}\left(\mathbb{R}^{3}\right)$.

Now, we assume that $\mathbf{w} \in \mathrm{L}^{2}(m)$ for some $m>7 / 2$. Then $\mathbf{w}$ can be decomposed as

$$
\mathbf{w}(\xi)=\sum_{i=1}^{3} \beta_{i} \mathbf{f}_{i}(\xi)+\sum_{i=1}^{3} \gamma_{i} \mathbf{g}_{i}(\xi)+\sum_{(i j) \in S} \zeta_{i j} \mathbf{h}_{i j}(\xi)+\tilde{\mathbf{w}}(\xi),
$$

where the coefficients $\beta_{i}$ are defined in (38) and $\gamma_{i}, \zeta_{i j}$ in (49). The velocity field $\mathbf{v}$ associated to $\mathbf{w}$ has a similar decomposition:

$$
\mathbf{v}(\xi)=\sum_{i=1}^{3} \beta_{i} \mathbf{v}^{\mathbf{f}_{i}}(\xi)+\sum_{i=1}^{3} \gamma_{i} \mathbf{v}^{\mathbf{g}_{i}}(\xi)+\sum_{(i j) \in S} \zeta_{i j} \mathbf{v}^{\mathbf{h}_{i j}}(\xi)+\tilde{\mathbf{v}}(\xi),
$$

where $\tilde{\mathbf{v}}$ is obtained from $\tilde{\mathbf{w}}$ via the Biot-Savart law (10). In view of (38) and $(\sqrt[66]{ })$, it is clear that $\beta_{i}=0$ for $i=1,2,3$ if and only if

$$
\int_{\mathbb{R}^{3}} \xi_{i} w_{j}(\xi) \mathrm{d} \xi=0 \quad \text { for all } i, j \in\{1,2,3\} .
$$

On the other hand, it follows from (49) and (67) that $\zeta_{i j}=0$ for all $(i j) \in S$ if and only if

$$
\begin{array}{ll}
M_{22}^{1}=M_{33}^{1}=-2 M_{12}^{2}=-2 M_{13}^{3}, & M_{11}^{1}=M_{22}^{2}=M_{33}^{3}=0, \\
M_{11}^{2}=M_{33}^{2}=-2 M_{12}^{1}=-2 M_{23}^{3}, & M_{23}^{1}=M_{13}^{2}=M_{12}^{3}=0 \\
M_{11}^{3}=M_{22}^{3}=-2 M_{13}^{1}=-2 M_{23}^{2}, &
\end{array}
$$

where

$$
M_{j k}^{i}=\int_{\mathbb{R}^{3}} \xi_{j} \xi_{k} w_{i}(\xi) \mathrm{d} \xi=M_{k j}^{i}, \quad i, j, k \in\{1,2,3\} .
$$

If in addition $\gamma_{i}=0$ for $i=1,2,3$, then $M_{j k}^{i}=0$ for all $i, j, k \in\{1,2,3\}$.

The main result of this section is the following estimate for the velocity field in terms of the vorticity: 
Proposition B.1 Let $\mathbf{w} \in \mathrm{L}^{2}(m)$ for some $m \geq 0$, and let $\mathbf{v}$ be the velocity field obtained from $\mathbf{w}$ via the Biot-Savart law (10). Define the coefficients $\beta_{i}$ by (38) and $\zeta_{i j}$ by (49). Assume that either

1) $0 \leq m<3 / 2$, or

2) $3 / 2<m<5 / 2$, or

3) $5 / 2<m<7 / 2$ and $\beta_{i}=0$ for $i=1,2,3$, or

4) $7 / 2<m<9 / 2, \beta_{i}=0$ for $i=1,2,3$, and $\zeta_{i j}=0$ for $(i j) \in S$.

Then there exists $C>0$ such that

$$
\left|\rho^{m} \mathbf{v}\right|_{6} \leq C\left|\rho^{m} \mathbf{w}\right|_{2},
$$

where $\rho(\xi)=1+|\xi|$.

Remark. More generally, Proposition B.1 holds for any (not necessarily divergence free) vector field $\mathbf{w}$ satisfying $\rho^{m} \mathbf{w} \in L^{2}\left(\mathbb{R}^{3}\right)^{3}$, provided that either

1) $0 \leq m<3 / 2$, or

2) $3 / 2<m<5 / 2$ and (65) holds, or

3) $5 / 2<m<7 / 2$ and (65), (71) hold, or

4) $7 / 2<m<9 / 2$ and (65), (71), (72) hold.

Roughly speaking, the result means that $\mathbf{v}(\xi)$ decays like $|\xi|^{-m-\frac{1}{2}}$ as $|\xi| \rightarrow \infty$.

The proof of Proposition B.1 is naturally divided into four steps. In the case 1), the bound (73) is a direct consequence of (22) and of the following weighted Hardy-LittlewoodSobolev inequality:

Lemma B.2 If $-1 / 2<m<3 / 2$ and

$$
u(\xi)=\int_{\mathbb{R}^{3}} \frac{\omega(\eta)}{|\xi-\eta|^{2}} \mathrm{~d} \eta, \quad \xi \in \mathbb{R}^{3},
$$

then $\left|\rho^{m} u\right|_{6} \leq C\left|\rho^{m} \omega\right|_{2}$.

Proof: We use the dyadic decomposition

$$
\mathbb{R}^{3}=\bigcup_{j=0}^{\infty} B_{j}
$$

where $B_{0}=\left\{\xi \in \mathbb{R}^{3}|| \xi \mid \leq 1\right\}$ and $B_{j}=\left\{\xi \in \mathbb{R}^{3}\left|2^{j-1}<\right| \xi \mid \leq 2^{j}\right\}$ for $j \in \mathbb{N}^{*}$. Let $u_{i}=u \mathbf{1}_{B_{i}}$ and $\omega_{i}=\omega \mathbf{1}_{B_{i}}, i \in \mathbb{N}$. Clearly $u_{i}=\sum_{j \in \mathbb{N}} \Delta_{i j}$, where

$$
\Delta_{i j}(\xi)=\mathbf{1}_{B_{i}}(\xi) \int_{B_{j}} \frac{\omega_{j}(\eta)}{|\xi-\eta|^{2}} \mathrm{~d} \eta .
$$

If $|i-j| \leq 1$, it follows from (11) that $\left|\Delta_{i j}\right|_{6} \leq C\left|\omega_{j}\right|_{2}$. If $|i-j| \geq 2$, Young's inequality implies that $\left|\Delta_{i j}\right|_{6} \leq M_{1}^{3 / 4} M_{2}^{1 / 4}\left|\omega_{j}\right|_{2}$, where

$$
M_{1}=\sup _{\xi \in B_{i}}\left(\int_{B_{j}} \frac{1}{|\xi-\eta|^{3}} \mathrm{~d} \eta\right)^{2 / 3}, \quad M_{2}=\sup _{\eta \in B_{j}}\left(\int_{B_{i}} \frac{1}{|\xi-\eta|^{3}} \mathrm{~d} \xi\right)^{2 / 3} .
$$


If $i \geq j+2$, then $|\xi-\eta| \geq|\xi|-|\eta| \geq 2^{i-1}-2^{j} \geq 2^{i-2}$ for all $\xi \in B_{i}, \eta \in B_{j}$. Thus $M_{1} \leq C 2^{-2 i} \mu\left(B_{j}\right)^{2 / 3} \leq C 2^{-2(i-j)}$ and $M_{2} \leq C 2^{-2 i} \mu\left(B_{i}\right)^{2 / 3} \leq C$ for some $C>0$ independent of $i, j$, hence $\left|\Delta_{i j}\right|_{6} \leq C 2^{-\frac{3}{2}(i-j)}\left|\omega_{j}\right|_{2}$. If $j \geq i+2$, then $|\xi-\eta| \geq 2^{j-2}$ for all $\xi \in B_{i}, \eta \in B_{j}$, and a similar calculation shows that $\left|\Delta_{i j}\right|_{6} \leq C 2^{-\frac{1}{2}(j-i)}\left|\omega_{j}\right|_{2}$. Summarizing, we have shown that

$$
\left|u_{i}\right|_{6} \leq C \sum_{j \in \mathbb{N}} K_{i j}\left|\omega_{j}\right|_{2}, \quad i \in \mathbb{N}
$$

where $K_{i j}=2^{-|i-j|-\frac{1}{2}(i-j)}$. Now, by definition of the sets $B_{i}$, we have $\left|\rho^{m} u_{i}\right| \leq C 2^{m i}\left|u_{i}\right|$ and $\left|\rho^{m} \omega_{j}\right| \geq C 2^{m j}\left|\omega_{j}\right|$ for all $i, j \in \mathbb{N}$. It follows that

$$
\left|\rho^{m} u_{i}\right|_{6} \leq C \sum_{j \in \mathbb{N}} K_{i j}^{(m)}\left|\rho^{m} \omega_{j}\right|_{2}, \quad i \in \mathbb{N}
$$

where $K_{i j}^{(m)}=2^{-|i-j|+\left(m-\frac{1}{2}\right)(i-j)}$. In particular, $\left|K_{i j}^{(m)}\right| \leq 2^{-\alpha|i-j|}$ for some $\alpha>0$, hence $K^{(m)}$ defines a bounded linear operator from $\ell^{2}(\mathbb{N})$ into $\ell^{6}(\mathbb{N})$. This concludes the proof.

QED

To prove (73) in the remaining cases, we also need the following variant of Lemma B.2:

Lemma B.3 If $-\frac{1}{2}<m<\frac{1}{2}$ and

$$
u(\xi)=\int_{\mathbb{R}^{3}} \frac{\omega(\eta)}{|\xi-\eta|} \mathrm{d} \eta, \quad \xi \in \mathbb{R}^{3},
$$

then $\left|\rho^{m} u\right|_{6} \leq C\left|\rho^{m+1} \omega\right|_{2}$.

Proof: We use the same notations as in the preceding proof. If $|i-j| \leq 1$, it follows from the Hardy-Littlewood-Sobolev inequality that $\left|\Delta_{i j}\right| \leq C\left|\omega_{j}\right|_{6 / 5}$. By Hölder, $\left|\omega_{j}\right|_{6 / 5} \leq C \mu\left(B_{j}\right)^{1 / 3}\left|\omega_{j}\right|_{2} \leq C 2^{j}\left|\omega_{j}\right|_{2}$, hence $\left|\Delta_{i j}\right|_{6} \leq C 2^{j}\left|\omega_{j}\right|_{2}$. If $|i-j| \geq 2$, then $\left|\Delta_{i j}\right|_{6} \leq N_{1}^{3 / 4} N_{2}^{1 / 4}\left|\omega_{j}\right|_{2}$, where

$$
N_{1}=\sup _{\xi \in B_{i}}\left(\int_{B_{j}} \frac{1}{|\xi-\eta|^{3 / 2}} \mathrm{~d} \eta\right)^{2 / 3}, \quad N_{2}=\sup _{\eta \in B_{j}}\left(\int_{B_{i}} \frac{1}{|\xi-\eta|^{3 / 2}} \mathrm{~d} \xi\right)^{2 / 3} .
$$

Proceeding as above, we deduce that $\left|\Delta_{i j}\right|_{6} \leq C 2^{-\frac{1}{2}|i-j|} 2^{j}\left|\omega_{j}\right|_{2}$ for all $i, j \in \mathbb{N}$. It follows that

$$
\left|\rho^{m} u_{i}\right|_{6} \leq C \sum_{j \in \mathbb{N}} \tilde{K}_{i j}^{(m)}\left|\rho^{m+1} \omega_{j}\right|_{2}
$$

where $\tilde{K}_{i j}^{(m)}=2^{-\frac{1}{2}|i-j|+m(i-j)}$. Thus, if $|m|<1 / 2, \tilde{K}^{(m)}$ defines a bounded linear operator from $\ell^{2}(\mathbb{N})$ into $\ell^{6}(\mathbb{N})$.

QED

We are now ready to prove Proposition B.1 in the case 2). If $3 / 2<m<5 / 2$ and $\mathbf{w} \in \mathrm{L}^{2}(m)$, then $\mathbf{w}$ is integrable and (65) holds. As a consequence, we can rewrite (22) in the form

$$
v_{i}(\xi)=-\frac{1}{4 \pi} \sum_{j, k=1}^{3} \epsilon_{i j k} \int_{\mathbb{R}^{3}}\left(\frac{\xi_{j}-\eta_{j}}{|\xi-\eta|^{3}}-\frac{\xi_{j}}{|\xi|^{3}}\right) w_{k}(\eta) \mathrm{d} \eta
$$


where $\epsilon_{i j k}=\operatorname{sign}(\sigma)$ if $(i j k)$ is a permutation $\sigma$ of (123), and $\epsilon_{i j k}=0$ otherwise. Using the identity

$$
|\xi|^{3}\left(\xi_{j}-\eta_{j}\right)-|\xi-\eta|^{3} \xi_{j}=\left(\xi_{j}-\eta_{j}\right)|\xi|^{2}(|\xi|-|\xi-\eta|)+|\xi-\eta|\left(2 \xi_{j}(\xi \cdot \eta)-\eta_{j}|\xi|^{2}-\xi_{j}|\eta|^{2}\right)
$$

we obtain

$$
\begin{aligned}
\left.|| \xi\right|^{3}\left(\xi_{j}-\eta_{j}\right)-|\xi-\eta|^{3} \xi_{j} \mid & \leq C|\xi-\eta||\xi||\eta|(|\xi|+|\eta|) \\
& \leq C\left(|\xi-\eta||\xi|^{2}|\eta|+|\xi-\eta|^{2}|\xi||\eta|\right)
\end{aligned}
$$

Thus, it follows from (74) that $|\mathbf{v}(\xi)| \leq C(f(\xi)+g(\xi))$, where

$$
f(\xi)=\frac{1}{|\xi|} \int_{\mathbb{R}^{3}} \frac{|\eta||\mathbf{w}(\eta)|}{|\xi-\eta|^{2}} \mathrm{~d} \eta, \quad g(\xi)=\frac{1}{|\xi|^{2}} \int_{\mathbb{R}^{3}} \frac{|\eta||\mathbf{w}(\eta)|}{|\xi-\eta|} \mathrm{d} \eta .
$$

Since we already know that $|\mathbf{v}|_{6} \leq C|\mathbf{w}|_{2}$, it is sufficient to bound $\left|\rho_{0}^{m} \mathbf{v}\right|_{6}$, where $\rho_{0}(\xi)=$ $|\xi| \mathbf{1}_{\{|\xi| \geq 1\}}$. Applying Lemma B.2 with $u(\xi)=|\xi| f(\xi)$ and $\omega(\eta)=|\eta||\mathbf{w}(\eta)|$, we find $\left|\rho_{0}^{m} f\right|_{6} \leq\left|\rho^{m-1} u\right|_{6} \leq C\left|\rho^{m-1} \omega\right|_{2} \leq C\left|\rho^{m} \mathbf{w}\right|_{2}$. Similarly, applying Lemma B.3 with $u(\xi)=$ $|\xi|^{2} g(\xi)$ and $\omega(\eta)=|\eta||\mathbf{w}(\eta)|$, we find $\left|\rho_{0}^{m} g\right|_{6} \leq C\left|\rho^{m-2} u\right|_{6} \leq C\left|\rho^{m-1} \omega\right|_{2} \leq C\left|\rho^{m} \mathbf{w}\right|_{2}$. Summarizing, we have shown that $\left|\rho^{m} \mathbf{v}\right|_{6} \leq C\left|\rho^{m} \mathbf{w}\right|_{2}$ if $3 / 2<m<5 / 2$.

We next assume that $5 / 2<m<7 / 2$ and that $\beta_{i}=0$ for $i=1,2,3$. As was already observed, this implies that (71) holds. Thus (74) can be written in the form

$$
v_{i}(\xi)=-\frac{1}{4 \pi} \sum_{j, k=1}^{3} \epsilon_{i j k} \int_{\mathbb{R}^{3}}\left(\frac{\xi_{j}-\eta_{j}}{|\xi-\eta|^{3}}-\frac{\xi_{j}-\eta_{j}}{|\xi|^{3}}-\frac{3(\xi \cdot \eta) \xi_{j}}{|\xi|^{5}}\right) w_{k}(\eta) \mathrm{d} \eta \text {. }
$$

To bound the right-hand side, we observe that

$$
\begin{aligned}
|\xi|^{5}\left(\xi_{j}-\eta_{j}\right) & -|\xi|^{2}|\xi-\eta|^{3}\left(\xi_{j}-\eta_{j}\right)-3|\xi-\eta|^{3}(\xi \cdot \eta) \xi_{j} \\
& =\left(\xi_{j}-\eta_{j}\right)|\xi|^{2}\left(|\xi|^{3}-|\xi-\eta|^{3}-3|\xi|(\xi \cdot \eta)\right) \\
& +3(\xi \cdot \eta)\left(|\xi|^{3}\left(\xi_{j}-\eta_{j}\right)-|\xi-\eta|^{3} \xi_{j}\right)
\end{aligned}
$$

We claim that

$$
\left.|| \xi\right|^{3}-|\xi-\eta|^{3}-\left.3|\xi|(\xi \cdot \eta)|\leq C| \eta\right|^{2}(|\xi|+|\eta|) .
$$

Indeed, this follows from the identity

$$
\begin{aligned}
|\xi|^{3}-|\xi-\eta|^{3}-3|\xi|(\xi \cdot \eta) & =2(|\xi-\eta|-|\xi|)(\xi \cdot \eta)-|\xi-\eta||\eta|^{2} \\
& -|\xi|\left(|\xi||\xi-\eta|+(\xi \cdot \eta)-|\xi|^{2}\right)
\end{aligned}
$$

and from the bound

$$
0 \leq|\xi||\xi-\eta|+(\xi \cdot \eta)-|\xi|^{2} \leq \frac{1}{2}|\eta|^{2},
$$

which is easily proved by setting $\eta=\xi-\zeta, \zeta \in \mathbb{R}^{3}$.

Using (77) together with (75), (78), we obtain

$$
\begin{aligned}
\left.|| \xi\right|^{5}\left(\xi_{j}-\eta_{j}\right)-|\xi|^{2}|\xi-\eta|^{3} & \left(\xi_{j}-\eta_{j}\right)-3|\xi-\eta|^{3}(\xi \cdot \eta) \xi_{j} \mid \\
& \leq C|\xi-\eta||\xi|^{2}|\eta|^{2}(|\xi|+|\eta|) \\
& \leq C\left(|\xi-\eta||\xi|^{3}|\eta|^{2}+|\xi-\eta|^{2}|\xi|^{2}|\eta|^{2}\right)
\end{aligned}
$$


Thus, it follows from (76) that $|\mathbf{v}(\xi)| \leq C(f(\xi)+g(\xi))$, where

$$
f(\xi)=\frac{1}{|\xi|^{2}} \int_{\mathbb{R}^{3}} \frac{|\eta|^{2}|\mathbf{w}(\eta)|}{|\xi-\eta|^{2}} \mathrm{~d} \eta, \quad g(\xi)=\frac{1}{|\xi|^{3}} \int_{\mathbb{R}^{3}} \frac{|\eta|^{2}|\mathbf{w}(\eta)|}{|\xi-\eta|} \mathrm{d} \eta .
$$

Applying Lemmas B.2 and B.3 as in the previous case, we easily obtain $\left|\rho_{0}^{m} f\right|_{6}+\left|\rho_{0}^{m} g\right|_{6} \leq$ $C\left|\rho^{m} \mathbf{w}\right|_{2}$. This prove (73) in the case 3).

Finally, we assume that $7 / 2<m<9 / 2$ and that $\beta_{i}=0$ for $i=1,2,3, \zeta_{i j}=0$ for $(i j) \in S$. As we already remarked, this implies that (72) holds. Using (72), it is straightforward (but somewhat tedious) to verify that

$$
\sum_{j, k=1}^{3} \epsilon_{i j k} \int_{\mathbb{R}^{3}}\left(2|\xi|^{2}(\xi \cdot \eta) \eta_{j}+\xi_{j}\left(|\xi|^{2}|\eta|^{2}-5(\xi \cdot \eta)^{2}\right)\right) w_{k}(\eta) \mathrm{d} \eta=0, \quad i \in \mathbb{N} .
$$

Assuming (80), we can rewrite $(\sqrt{76})$ in the form

$$
v_{i}(\xi)=-\frac{1}{4 \pi} \sum_{j, k=1}^{3} \epsilon_{i j k} \int_{\mathbb{R}^{3}} \frac{A_{j}(\xi, \eta) w_{k}(\eta)}{|\xi-\eta|^{3}|\xi|^{7}} \mathrm{~d} \eta
$$

where

$$
\begin{aligned}
A_{j}(\xi, \eta) & =|\xi|^{7}\left(\xi_{j}-\eta_{j}\right)-|\xi|^{4}|\xi-\eta|^{3}\left(\xi_{j}-\eta_{j}\right) \\
& -3|\xi|^{2}|\xi-\eta|^{3}(\xi \cdot \eta)\left(\xi_{j}-\eta_{j}\right)-\frac{3}{2} \xi_{j}|\xi-\eta|^{3}\left(5(\xi \cdot \eta)^{2}-|\xi|^{2}|\eta|^{2}\right) .
\end{aligned}
$$

We claim that

$$
\left|A_{j}\right| \leq C|\xi-\eta||\xi|^{3}|\eta|^{3}(|\xi|+|\eta|) \leq C\left(|\xi-\eta||\xi|^{4}|\eta|^{3}+|\xi-\eta|^{2}|\xi|^{3}|\eta|^{3}\right) .
$$

Assuming (82) for the moment, we deduce from (81) that $|\mathbf{v}(\xi)| \leq C(f(\xi)+g(\xi))$, where

$$
f(\xi)=\frac{1}{|\xi|^{3}} \int_{\mathbb{R}^{3}} \frac{|\eta|^{3}|\mathbf{w}(\eta)|}{|\xi-\eta|^{2}} \mathrm{~d} \eta, \quad g(\xi)=\frac{1}{|\xi|^{4}} \int_{\mathbb{R}^{3}} \frac{|\eta|^{3}|\mathbf{w}(\eta)|}{|\xi-\eta|} \mathrm{d} \eta .
$$

Applying Lemmas B.2 and B.3 again, we obtain $\left|\rho_{0}^{m} f\right|_{6}+\left|\rho_{0}^{m} g\right|_{6} \leq C\left|\rho^{m} \mathbf{w}\right|_{2}$. This proves (73) in the case 4).

It remains to establish (82). We first remark that $A_{j}=\left(\xi_{j}-\eta_{j}\right)|\xi|^{2} B+C_{j}$, where

$$
\begin{aligned}
B & =|\xi|^{5}-|\xi|^{2}|\xi-\eta|^{3}-3|\xi-\eta|^{3}(\xi \cdot \eta)-\frac{3}{2}|\xi|\left(5(\xi \cdot \eta)^{2}-|\xi|^{2}|\eta|^{2}\right) \\
C_{j} & =\frac{3}{2}\left(|\xi|^{3}\left(\xi_{j}-\eta_{j}\right)-\xi_{j}|\xi-\eta|^{3}\right)\left(5(\xi \cdot \eta)^{2}-|\xi|^{2}|\eta|^{2}\right) .
\end{aligned}
$$

In view of $(\overline{75})$, it is clear that $\left|C_{j}\right| \leq C|\xi-\eta||\xi|^{3}|\eta|^{3}(|\xi|+|\eta|)$. Thus, it is sufficient to show that

$$
|B| \leq C|\xi||\eta|^{3}(|\xi|+|\eta|) .
$$

To this end, we remark that

$$
B=|\xi| D+(\xi \cdot \eta)\left(|\xi|^{3}-|\xi-\eta|^{3}-3|\xi|(\xi \cdot \eta)\right)
$$




$$
+(|\xi-\eta|-|\xi|)\left(4(\xi \cdot \eta)^{2}-|\xi|^{2}|\eta|^{2}\right)-2|\eta|^{2}(\xi \cdot \eta)|\xi-\eta|
$$

where

$$
\begin{aligned}
D & =|\xi|^{4}-|\xi|^{2}(\xi \cdot \eta)+\frac{1}{2}|\xi|^{2}|\eta|^{2}-\frac{1}{2}(\xi \cdot \eta)^{2}-|\xi|^{3}|\xi-\eta| \\
& =\frac{1}{2}\left(|\xi||\xi-\eta|+(\xi \cdot \eta)-|\xi|^{2}\right)(|\xi|(|\xi-\eta|-|\xi|)-(\xi \cdot \eta)) .
\end{aligned}
$$

Using (79), we find $|D| \leq \frac{1}{2}|\xi||\eta|^{3}$. Inserting this bound into (84) and using (78), we obtain (83). This concludes the proof of Proposition B.1.

QED

We conclude with two corollaries which are used in the preceding sections.

Corollary B.4 Assume that $\mathbf{w} \in \mathrm{L}^{2}(m)$ for some $m>\frac{5}{2}$, and denote by $\mathbf{v}$ the velocity field obtained from $\mathbf{w}$ via the Biot-Savart law (10). Then $\mathbf{v} \in L^{1}\left(\mathbb{R}^{3}\right)^{3}$ if and only if $\beta_{i}=0$ for $i=1,2,3$, and in this case $\int_{\mathbb{R}^{3}} v_{i}(\xi) \mathrm{d} \xi=0$ for $i=1,2,3$.

Proof: Without loss of generality, we assume that $5 / 2<m<7 / 2$. For any $\mathbf{w} \in \mathrm{L}^{2}(m)$, we have the decomposition

$$
\mathbf{w}=\sum_{i=1}^{3} \beta_{i} \mathbf{f}_{i}+\tilde{\mathbf{w}}, \quad \mathbf{v}=\sum_{i=1}^{3} \beta_{i} \mathbf{v}^{\mathbf{f}_{i}}+\tilde{\mathbf{v}},
$$

where the coefficients $\beta_{i}$ are defined in (38). Then the remainder $\tilde{\mathbf{w}} \in \mathrm{L}^{2}(m)$ fulfills the moment conditions (71). By Proposition B.1, the corresponding velocity field satisfies $\rho^{m} \tilde{\mathbf{v}} \in L^{6}\left(\mathbb{R}^{3}\right)^{3}$, hence $\tilde{\mathbf{v}} \in \mathrm{L}^{1}\left(\mathbb{R}^{3}\right)$. On the other hand, it is easy to verify that $\sum \beta_{i} \mathbf{v}^{\mathbf{f}_{i}} \in$ $\mathrm{L}^{1}\left(\mathbb{R}^{3}\right)$ if and only if $\beta_{i}=0$ for $i=1,2,3$. Thus $\mathbf{v} \in \mathrm{L}^{1}\left(\mathbb{R}^{3}\right)$ if and only if $\beta_{1}=\beta_{2}=\beta_{3}=0$. In this case, $\int_{\mathbb{R}^{3}} v_{i}(\xi) \mathrm{d} \xi=0$ for $i=1,2,3$ because $\operatorname{div} \mathbf{v}=0$, see (65).

QED

Corollary B.5 Assume that $\mathbf{w} \in \mathrm{L}^{2}(m)$ for some $m>\frac{7}{2}$, and denote by $\mathbf{v}$ the velocity field obtained from $\mathbf{w}$ via the Biot-Savart law (19). Then $\rho \mathbf{v} \in L^{1}\left(\mathbb{R}^{3}\right)^{3}$ if and only if $\beta_{i}=0$ for $i=1,2,3$ and $\zeta_{i j}=0$ for $(i j) \in S$. In this case, the matrix $\left(b_{k \ell}\right)$ defined by

$$
b_{k \ell}=\int_{\mathbb{R}^{3}} \xi_{k} v_{\ell}(\xi) \mathrm{d} \xi, \quad k, \ell \in\{1,2,3\},
$$

is skew-symmetric, and $b_{12}=\gamma_{3}, b_{23}=\gamma_{1}, b_{31}=\gamma_{2}$.

Proof: Without loss of generality, we assume that $7 / 2<m<9 / 2$. If $\mathbf{w} \in \mathrm{L}^{2}(m)$, then $\mathbf{w}$ and $\mathbf{v}$ can be decomposed according to (69), (70). By construction, the remainder $\tilde{\mathbf{w}} \in$ $\mathrm{L}^{2}(m)$ has vanishing first-order and second-order moments. Applying Proposition B.1, we deduce that the corresponding velocity field satisfies $\rho^{m} \tilde{\mathbf{v}} \in L^{6}\left(\mathbb{R}^{3}\right)^{3}$, hence $\rho \tilde{\mathbf{v}} \in L^{1}\left(\mathbb{R}^{3}\right)^{3}$. Therefore, using the expressions above of $\mathbf{f}_{i}, \mathbf{g}_{i}$, and $\mathbf{h}_{i j}$, it is not difficult to show that $\rho \mathbf{v} \in L^{1}\left(\mathbb{R}^{3}\right)^{3}$ if and only if $\beta_{i}=0$ for $i=1,2,3$ and $\zeta_{i j}=0$ for $(i j) \in S$. In this case, the matrix $\left(b_{k \ell}\right)$ defined by $(85)$ is skew-symmetric because div $\mathbf{v}=0$, see (66).

Assume now that $\rho \mathbf{v} \in L^{1}\left(\mathbb{R}^{3}\right)^{3}$, and consider the vector field $\mathbf{A}(\xi)=\mathbf{q}_{3}(\xi) \wedge \mathbf{v}(\xi)$, where $\mathbf{q}_{3}$ is defined in (45)). Then $\mathbf{A} \in L^{3 / 2}\left(\mathbb{R}^{3}\right)^{3}$ and $\operatorname{div} \mathbf{A}=\mathbf{p}_{3} \cdot \mathbf{v}-\mathbf{q}_{3} \cdot \mathbf{w}$, where $\mathbf{p}_{3}=\operatorname{rot} \mathbf{q}_{3}$ is defined in (36). It follows that

$$
0=\int_{\mathbb{R}^{3}} \operatorname{div} \mathbf{A} \mathrm{d} \xi=\int_{\mathbb{R}^{3}}\left(\mathbf{p}_{3} \cdot \mathbf{v}-\mathbf{q}_{3} \cdot \mathbf{w}\right) \mathrm{d} \xi=b_{12}-\gamma_{3},
$$

in view of (36), (85), and (49). The relations $b_{23}=\gamma_{1}$ and $b_{31}=\gamma_{2}$ are proved in a similar way.

QED 


\section{References}

[1] L. Brandolese. On the localization of symmetric and asymmetric solutions of the Navier-Stokes equations in $\mathbb{R}^{n}$. To appear in C. R. Acad. Sci. Paris, Série I, 2001.

[2] J. Bricmont and A. Kupiainen. Stable non-Gaussian diffusive profiles. Nonlinear Anal., 26(3):583-593, 1996.

[3] M. Cannone and F. Planchon. Self-similar solutions for Navier-Stokes equations in $\mathbb{R}^{3}$. Commun. Partial Differ. Equations, 21(1-2):179-193, 1996.

[4] A. Carpio. Asymptotic behavior for the vorticity equations in dimensions two and three. Comm. Partial Differential Equations, 19(5-6):827-872, 1994.

[5] A. Carpio. Large-time behavior in incompressible navier-stokes equations. SIAM J. Math. Anal., 27(2):449-475, 1996.

[6] J.-P. Eckmann and C. E. Wayne. Non-linear stability analysis of higher-order dissipative partial differential equations. Math. Phys. Electron. J., 4:Paper 3, 20 pp. (electronic), 1998.

[7] J.-P. Eckmann, C. E. Wayne, and P. Wittwer. Geometric stability analysis for periodic solutions of the Swift-Hohenberg equation. Comm. Math. Phys., 190(1):173-211, 1997.

[8] M. Escobedo, O. Kavian, and H. Matano. Large time behavior of solutions of a dissipative semilinear heat equation. Comm. Partial Differential Equations, 20(78):1427-1452, 1995.

[9] Y. Fujigaki and T. Miyakawa. Asymptotic profiles of nonstationary incompressible Navier-Stokes flows in $\mathbb{R}^{n}$. Preprint, 2000.

[10] V. A. Galaktionov and J. L. Vázquez. Asymptotic behaviour of nonlinear parabolic equations with critical exponents. A dynamical systems approach. J. Funct. Anal., 100(2):435-462, 1991.

[11] Th. Gallay and G. Raugel. Scaling variables and asymptotic expansions in damped wave equations. J. Differential Equations, 150(1):42-97, 1998.

[12] Th. Gallay and G. Raugel. Scaling variables and stability of hyperbolic fronts. SIAM J. Math. Anal., 32(1):1-29 (electronic), 2000.

[13] Th. Gallay and C. E. Wayne. Invariant manifolds and the long-time asymptotics of the Navier-Stokes and vorticity equations on $\mathbb{R}^{2}$. Preprint, 2001.

[14] Y. Giga and T. Miyakawa. Navier-Stokes flow in $\mathbb{R}^{3}$ with measures as initial vorticity and Morrey spaces. Comm. Partial Differential Equations, 14(5):577-618, 1989.

[15] T. Kato. Strong $L^{p}$-solutions of the Navier-Stokes equation in $\mathbb{R}^{m}$, with applications to weak solutions. Math. Z., 187(4):471-480, 1984. 
[16] O. Kavian. Remarks on the large time behaviour of a nonlinear diffusion equation. Ann. Inst. H. Poincaré Anal. Non Linéaire, 4(5):423-452, 1987.

[17] T. Miyakawa and M. E. Schonbek. On optimal decay rates for weak solutions to the Navier-Stokes equations in $\mathbb{R}^{n}$. Preprint, 2000.

[18] M. E. Schonbek. $L^{2}$ decay for weak solutions of the Navier- Stokes equations. Arch. Rational Mech. Anal., 88(3):209-222, 1985.

[19] M. E. Schonbek. Large time behaviour of solutions to the Navier-Stokes equations. Comm. Partial Differential Equations, 11(7):733-763, 1986.

[20] M. E. Schonbek. Lower bounds of rates of decay for solutions to the Navier-Stokes equations. J. Amer. Math. Soc., 4(3):423-449, 1991.

[21] M. E. Schonbek. Asymptotic behavior of solutions to the three-dimensional NavierStokes equations. Indiana Univ. Math. J., 41(3):809-823, 1992.

[22] E. M. Stein. Singular integrals and differentiability properties of functions. Princeton University Press, Princeton, N.J., 1970. Princeton Mathematical Series, No. 30.

[23] C. E. Wayne. Invariant manifolds for parabolic partial differential equations on unbounded domains. Arch. Rational Mech. Anal., 138(3):279-306, 1997.

[24] M. Wiegner. Decay results for weak solutions of the Navier-Stokes equations on $\mathbb{R}^{n}$. J. Lond. Math. Soc., II. Ser., 35:303-313, 1987.

[25] E. Zuazua. Weakly nonlinear large time behavior in scalar convection-diffusion equations. Differential Integral Equations, 6(6):1481-1491, 1993. 